\title{
Embodied language in first- and second-language speakers: Neural correlates of processing motor verbs
}

\author{
Sophie De Grauwe ${ }^{\text {a,* }}$, Roel M. Willems ${ }^{\text {a,b }}$, Shirley-Ann Rueschemeyer ${ }^{a}$, Kristin Lemhöfer ${ }^{\text {, }}$, \\ Herbert Schriefers ${ }^{\text {a }}$ \\ ${ }^{a}$ Radboud University Nijmegen, Donders Institute for Brain, Cognition and Behaviour, Nijmegen, Netherlands \\ ${ }^{\mathrm{b}}$ Max Planck Institute for Psycholinguistics, Nijmegen, The Netherlands
}

\section{A R T I C L E I N F O}

\section{Article history:}

Received 11 June 2013

Received in revised form

22 January 2014

Accepted 3 February 2014

Available online 10 February 2014

Keywords:

Language

fMRI

Embodied

Bilingual

Action verb

\begin{abstract}
A B S T R A C T
The involvement of neural motor and sensory systems in the processing of language has so far mainly been studied in native (L1) speakers. In an fMRI experiment, we investigated whether non-native (L2) semantic representations are rich enough to allow for activation in motor and somatosensory brain areas. German learners of Dutch and a control group of Dutch native speakers made lexical decisions about visually presented Dutch motor and non-motor verbs. Region-of-interest (ROI) and whole-brain analyses indicated that L2 speakers, like L1 speakers, showed significantly increased activation for simple motor compared to non-motor verbs in motor and somatosensory regions. This effect was not restricted to Dutch-German cognate verbs, but was also present for non-cognate verbs. These results indicate that L2 semantic representations are rich enough for motor-related activations to develop in motor and somatosensory areas.
\end{abstract}

(c) 2014 Elsevier Ltd. All rights reserved.

\section{Introduction}

During the last decade, the role of sensory and motor neural systems in the construction of linguistic meaning has received a great deal of attention. It has been postulated that the processing of action- and perception-related language leads to activation of the same brain areas as action and perception themselves. In other words, language is grounded in bodily action and perception, or 'embodied' (Barsalou, 2008; but see Mahon \& Caramazza, 2008). Most studies on language embodiment have focused on native (L1) speakers. In contrast, non-native (L2) speakers have hardly been investigated in this regard (see below). It has been claimed that L2 semantic representations are less developed than L1 semantic representations (Finkbeiner, Forster, Nicol, \& Nakamura, 2004 - for details, see below). When we extrapolate this to the question of language embodiment in L2 speakers, this implies that activation in action- and perception-related brain areas for L2 words may be absent or reduced compared to L1 words.

To our knowledge, the present study is the first functional magnetic resonance imaging (fMRI) study to investigate whether evidence of embodied grounding of language can be found with L2 speakers (but see Buchweitz, Shinkareva, Mason, Mitchell, \& Just,

\footnotetext{
* Correspondence to: Radboud Universiteit Nijmegen, Postbus 9104, 6500 HE Nijmegen, The Netherlands. Tel.: + 31243612634.

E-mail address: s.degrauwe@donders.ru.nl (S. De Grauwe).
}

2012, for some evidence indirectly related to embodied cognition in bilinguals). Our results will extend insights into embodiment effects to a new domain, i.e. L2 processing, and thus also shed more light on how non-native language is processed. For this, we will study both morphologically simple and complex verbs. In the remainder of this paper, the term 'embodiment effects' will be used as a shorthand for the occurrence of activations in actionand/or somatosensory-related brain areas in response to words with a motor-related meaning. With the term embodiment effects, we do not imply any commitment as to the source of these activations, i.e. whether they are a necessary part of semantic representations or a by-product of such representations (i.e. epiphenomenal).

\subsection{Embodiment effects with morphologically simple words}

Motor and/or premotor activations are reported in a variety of fMRI studies on action or motor verbs. Hauk, Johnsrude, and Pulvermüller (2004) investigated neural correlates of passively read face-, arm- and leg-related motor verbs (e.g., lick, pick and kick) and the corresponding actions executed by participants. When comparing motor verbs to baseline, they found somatotopically organized activation of motor and premotor cortex, and partial overlap of these with activations for face, arm and leg actions. These motor-related activations were interpreted as reflecting meaning representations of motor verbs. Somatotopic 
organization of activations in motor regions was also found by Raposo, Moss, Stamatakis, and Tyler (2009) when participants listened to arm- (e.g., grab) and leg-related (e.g., trample) motor verbs. Again, these activations partially overlapped with activations for arm and leg actions, respectively (but see also their results on idiomatic sentences in that same study; see also Kemmerer, Castillo, Talavage, Patterson, \& Wiley, 2008, for (partial) support for somatotopically organized activations). No such somatotopic organization was found by Postle, McMahon, Ashton, Meredith, and de Zubicaray (2008), although they did report pre-SMA activation when passively read motor verbs (leg-, arm- and mouth-related motor verbs taken together) were compared with non-motor nouns. This activation was interpreted as reflecting the retrieval of motor programs, with motor verbs serving as instructional cues.

Other regions commonly reported in neuroimaging studies on motor verbs are located in the parietal lobe, for example the anterior inferior parietal cortex (aIPC, associated with abstract somatosensory knowledge of actions - see Binder, Desai, Graves, \& Conant, 2009), or the parietal operculum (secondary somatosensory cortex, associated with finger stimulation - see Ruben et al., 2001). Noppeney, Josephs, Kiebel, Friston, and Price (2005) found activations in the aIPC for semantic decisions to motor verbs relative to non-motor words. In a visual lexical decision fMRI study, Rüschemeyer, Brass, and Friederici (2007) reported not only activations in left precentral gyrus and central sulcus as well as bilateral postcentral gyrus in response to German simple handrelated motor verbs (e.g., werfen 'throw') versus non-motor verbs (e.g., denken 'think'), but also in left parietal operculum (S2). In contrast, a comparison of these same motor and non-motor verbs embedded as stems in complex verbs with a non-motor meaning (e.g., werfen in entwerfen 'design' - denken in bedenken 'consider') revealed no activations in sensorimotor (i.e. (pre)motor or somatosensory) areas.

All these studies focused on the processing of motor verbs by L1 speakers (see Willems \& Casasanto, 2011, for an overview). It is unclear, though, whether L2 speakers display the same kind of embodiment effects as L1 speakers, as hardly any study has addressed this issue. A number of fMRI studies have looked into semantic processing in bilinguals (Chee, Hon, Lee, \& Soon, 2001; Illes et al., 1999; Isel, Baumgaertner, Thrän, Meisel, \& Büchel, 2010; Rüschemeyer, Zysset, \& Friederici, 2006; Wartenburger et al., 2003). However, none of these systematically manipulated motor-relatedness, for example by including a contrast between motor and non-motor words or between different types of motor words, thus precluding any conclusions as to the embodied nature of L2 semantic representations.

The only study in which language embodiment effects in L2 speakers were investigated is a behavioral study using a pictureverb matching task (Bergen, Lau, Narayan, Stojanovic, \& Wheeler, 2010). For both L1 and advanced L2 participants, judgment times were longer when the word and the picture referred to different actions performed with the same effector (mouth, hand or foot; e.g., run-kick) than when they referred to different actions performed with different effectors (e.g., run-drink). This suggests that words and pictures led to activation of the same sensorimotor circuits, causing interference when the same effector was involved.

Although most models of bilingual word processing do not speak to the presence or absence of embodiment effects in L2 speakers, their descriptions of L1 versus L2 processing allow us to derive hypotheses concerning embodiment effects in L2 speakers. Some models argue for shared semantic representations in L1 and L2, whereas other models claim that L2 semantic representations are less detailed than L1 semantic representations. The first position is taken by the Revised Hierarchical Model (RHM, Kroll \& Stewart, 1994) and the extended Bilingual Interactive Activation $(B I A+)$ Model (Dijkstra \& Van Heuven, 2002). In both models, access to word meanings is delayed in L2 compared to L1, but there is no difference in the semantic information accessed in L1 and L2, as semantic representations are shared across languages. Therefore, these models should predict no differences between L1 and L2 speakers with respect to embodiment effects: The same sensorimotor regions would be involved in L1 and L2 speakers, and the difference in speed of access to semantic representations would be too slight to be picked up by a method with such a limited temporal resolution as fMRI.

The second position, i.e. less detailed L2 semantic representations, is taken by the Sense Model (Finkbeiner et al., 2004). This model argues for (partially) overlapping distributed semantic representations for L1 and L2 words. L2 words are supposed to have "less rich" semantic representations, i.e. they may be associated with fewer senses than L1 words. Therefore, according to this model, embodiment effects might be reduced or even absent in L2 speakers.

\subsection{Embodiment effects with morphologically complex words: decomposition or holistic processing?}

Embodiment effects are mostly used to shed light on the question of language embodiment per se. However, they can also be used as a tool to investigate whether morphologically complex words are decomposed into their constituent parts or processed holistically during comprehension. As far as we know, this approach has so far only been used in Rüschemeyer et al.'s (2007) study on German as L1. In this study, morphologically complex derivations (i.e. words in which a stem is combined with a morpheme that changes the meaning of the stem) were included which were abstract and opaque: Their meaning as a whole was not related to the (motor-related) meaning of their parts. For example, the meaning of entwerfen ('design') is not semantically related to the meaning of its motor-related stem werfen ('throw'). Finding embodiment effects for these complex verbs would indicate that the meaning of the motor-related stems was accessed, i.e. that the opaque complex verbs were decomposed into prefix and (motor-related) stem. However, the results showed a significant interaction between complexity and motor-relatedness, with embodiment effects with simple motor versus non-motor verbs, but not with complex verbs with motor versus non-motor stem. This suggests that opaque complex verbs were processed holistically.

The processing of morphologically complex derivations has been studied extensively in behavioral experiments in L1 speakers. Often, morphological priming/lexical decision experiments are used to compare opaque complex words such as 'restrain' with transparent complex verbs such as 'reheat' (whose meaning as a whole is related to the meaning of their constituent parts). In such experiments, first, a prime word is presented (e.g., a complex verb), followed by the presentation of a target word (e.g., the stem of the complex verb prime). A lexical decision has to be made to the target word. With supraliminal, i.e. non-masked priming designs (in which the prime word is shown long enough to be perceived consciously), many studies find a dissociation between priming for transparent versus opaque conditions: Transparent conditions show facilitatory priming, while this is not the case for opaque conditions. This has been found for visual priming (visually presented primes and targets; English: Feldman \& Soltano, 1999; Feldman, Soltano, Pastizzo, \& Francis, 2004; Rastle, Davis, Marslen-Wilson, \& Tyler, 2000; Serbian: Feldman, Barac-Cikoja, \& Kostić, 2002; but see Smolka, Komlósi, \& Rösler, 2009 (German)) and cross-modal priming (auditorily presented primes and visual targets; English: Feldman et al., 2004; Gonnerman, Seidenberg, \& Andersen, 2007; Marslen-Wilson, Tyler, Waksler, \& Older, 1994; French: Longtin, Segui, \& Hallé, 2003; Dutch: Zwitserlood, Bolwiender, \& Drews, 2005; but see Luttmann, 
Zwitserlood, \& Bolte, 2011 (German)). ${ }^{1}$ These results suggest that transparent complex verbs are decomposed during word recognition, whereas opaque complex verbs are processed holistically.

To our knowledge, supraliminal (non-masked) priming/lexical decision studies on derivations have not yet been reported with L2 speakers. However, there are a few studies on L2 morphological processing in which other methods (such as masked priming) and/or other materials (such as inflections, e.g., walk-walked, or compounds, e.g., sleepwalk) were used. These studies have produced conflicting results. Some studies focusing on derivations have found evidence for more reliance of L2 speakers on holistic processing (Clahsen \& Neubauer, 2010; Silva \& Clahsen, 2008), whereas others have found no difference between L1 and L2 speakers (Diependaele, Duñabeitia, Morris, \& Keuleers, 2011; Portin \& Laine, 2001). Similarly, some studies on inflections have found evidence for more reliance of L2 speakers on holistic processing (Neubauer \& Clahsen, 2009; Silva \& Clahsen, 2008), whereas others have found more L2 reliance on decomposition (Lehtonen \& Laine, 2003; Portin \& Laine, 2001). Lemhöfer, Koester, and Schreuder (2011)'s study on noun compounds also found evidence for more L2 reliance on decomposition. Any direct comparison between these studies is complicated by differences between the tasks (masked priming vs. unprimed lexical decision) and/or materials (inflections vs. derivations vs. compounds), and by different combinations of languages used as L1 and L2, since differences in morphological processing in the $\mathrm{L} 2$ may be due to differences in (the morphological richness of) the L1 of the participants (see Basnight-Brown, Chen, Hua, Kostić, \& Feldman, 2007; Portin et al., 2008). Also, the contrast between transparent and opaque complex words was not specifically targeted by the studies discussed (except by Diependaele et al., 2011, in which masked priming was used). Therefore, it is difficult to reach conclusions with regard to the processing of opaque or transparent derived verbs by 22 speakers.

\subsection{The present study}

In this fMRI study, we focus on German advanced L2 speakers of Dutch. German and Dutch are highly related languages with a large number of cognates, i.e. words with similar form and meaning in the two languages. Cognates have been found to elicit faster reaction times than non-cognates in word recognition studies, the so-called cognate facilitation effect (e.g., Dijkstra, Miwa, Brummelhuis, Sappelli, \& Baayen, 2010). This effect indicates that, in L2 processing, there might be transfer from L1 to L2 through cognates: As semantic representations may be less firmly established in L2, they may be more easily accessible with cognates than non-cognates. To maximize our chances of finding embodiment effects in L2, we included cognate verbs. These were contrasted with non-cognate verbs, allowing us to find out whether potential embodiment effects with motor verbs are primarily mediated by L1-L2 transfer due to cognate status, or whether they are present for cognate and non-cognate verbs alike.

We were interested in two issues. Firstly, we wanted to find out whether, like L1 speakers, L2 speakers show embodiment effects with morphologically simple verbs. For this, we investigated whether Dutch simple motor verbs induce more activation in S2 and/or (pre)motor areas than Dutch simple non-motor verbs in German L2 speakers of Dutch and in Dutch L1 speakers. These simple verbs were either cognates (e.g., nemen (German: nehmen) 'take') or non-cognates (e.g.,

\footnotetext{
${ }^{1}$ In this overview of L1 morphological processing literature, we focus on studies including similar materials and methods as our study, i.e. morphologically complex derivations investigated with non-masked priming. Due to the lack of such studies in L2 processing, our L2 overview also includes other materials and/or methods.
}

gooien (German: werfen) 'throw'), to investigate the role of form overlap between L1-L2 translation pairs.

Secondly, we wanted to know whether L2 speakers decompose opaque complex verbs (in contrast with L1 speakers) or process them holistically (like L1 speakers). To investigate this, we compared the processing of Dutch opaque complex verbs with a motor stem with Dutch opaque complex verbs with a non-motor stem in German L2 speakers of Dutch and in Dutch L1 speakers. If opaque complex verbs with a motor stem (whose meaning as a whole is not motor-related) were found to induce more activation in S2 and/or (pre)motor areas than opaque complex verbs with a non-motor stem in L2 speakers, this would suggest that the meaning of the motor stem was accessed, i.e. that opaque complex verbs are decomposed. If no increased activation of S2 and/or (pre)motor areas was found, this would suggest that opaque complex verbs are processed holistically.

To investigate these questions, a 2 (Complexity: Simple vs. Complex $) \times 2$ (Cognate Status: Cognate vs. Non-Cognate $) \times 2$ (Motor-Relatedness: Motor vs. Non-Motor) design would have been ideal. However, the close relation between Dutch and German made it impossible to find enough non-cognate opaque complex verbs, leading to two empty cells in the design (see Table 1). Therefore, we opted for a combination of two $2 \times 2$ subdesigns. In the first one, the Simple Verb subdesign, only morphologically simple verbs were examined, with Cognate Status (Cognate vs. Non-Cognate) and Motor-Relatedness (Motor vs. Non-Motor) as factors. In the second one, the Cognate Verb subdesign, only cognate verbs were included, with Complexity (Simple vs. Complex) and Motor-Relatedness (Motor vs. Non-Motor) as factors. Adding the factor of Language (L1 vs. L2 speakers) enabled us to compare the two language groups, turning the subdesigns into $2 \times 2 \times 2$ designs.

The Simple Verb subdesign allowed us to investigate whether motor-related activations (i.e. higher activations for motor than for non-motor words) can be found with simple verbs not only in L1, but also in L2. We hypothesized that, since the L2 speakers were advanced speakers of Dutch, such activations would indeed become apparent. However, if L2 speakers' semantic representations are less rich than those of L1 speakers (Finkbeiner et al., 2004), motor-related activations in L2 speakers might be reduced compared to L1 speakers.

With the Cognate Verb subdesign, we first aimed at a replication of the interaction between Complexity and Motor-Relatedness reported by Rüschemeyer et al. (2007) for L1 speakers. For L2 speakers, this subdesign allowed us to investigate whether L2 speakers show a similar pattern as L1 speakers. In line with Rüschemeyer et al., we expected L1 speakers to show motor-related activation for simple verbs, but not for opaque complex verbs. The absence of motor-related activation for opaque complex verbs would be an indication that these verbs are not decomposed. As mentioned above, evidence on L2 morphological processing is mixed. If L2 speakers decompose opaque complex verbs, we should find motor-related activation for both simple and complex verbs. If they process opaque complex verbs holistically, we should find the same results as for L1 speakers, i.e. motor-related activation for simple motor verbs and no motor-related activation for opaque complex verbs containing motor stems.

\section{Table 1}

Design: A combination of two subdesigns.

\begin{tabular}{|c|c|c|}
\hline Cognate Verb & $\begin{array}{l}\text { Simple Verb } \\
\text { Motor } \\
\text { Non-Motor }\end{array}$ & $\begin{array}{l}\text { Opaque Complex Verb } \\
\text { Motor } \\
\text { Non-Motor }\end{array}$ \\
\hline Non-Cognate Verb & $\begin{array}{l}\text { Motor } \\
\text { Non-Motor }\end{array}$ & $\begin{array}{l}- \\
-\end{array}$ \\
\hline
\end{tabular}




\section{Method}

\subsection{Participants}

Twenty-two Dutch participants (L1 speakers) and 29 German advanced learners of Dutch (L2 speakers) initially took part. After participant exclusion (see below for details), $20 \mathrm{~L} 1$ and $18 \mathrm{~L} 2$ speakers remained for the final analyses. The remaining L1 speakers (16 females, 4 males) were aged 19-26 ( $M=21.95$, $\mathrm{SD}=2.31$ ). The remaining L2 speakers (10 females, 8 males) were aged $22-29$ $(M=24.44, S D=2.15)$. All participants signed a written consent form in accordance with the national legislation for human protection and the Helsinki Declaration of 1975 , revised in 2004.

L2 speakers, most of them German students at Radboud University Nijmegen, were recruited on the basis of the following criteria: They had German as their dominant language, had lived and/or studied in the Netherlands for at least 1.5 years, and used Dutch regularly for studies, work and/or private life. In order to determine their proficiency level in Dutch, they were required to take part in the online version of the Dutch LexTALE test, a vocabulary test using non-speeded visual lexical decision (Lemhöfer \& Broersma, 2012). Only L2 speakers with a minimum of $67.50 \%$ correct in this test were invited to participate in the fMRI experiment ( $M=78.89 \%$; $S D=8.03 \%$, range $67.50-96.25 \%)$. After participation in the fMRI experiment, L2 speakers were asked to fill out a language history questionnaire. The mean age of acquisition of Dutch was $19.94(\mathrm{SD}=2.88$ ). Participants were also asked to rate their experience with and their frequency of using Dutch (see Appendix A in Supplementary Materials).

L1 speakers, most of them students at Radboud University Nijmegen, had Dutch as their first and dominant language and had lived in the Netherlands from birth. All participants (L1 and L2 speakers) were right-handed and reported having no reading disorders.

\subsection{Materials}

Three categories of Dutch verbs were selected for this experiment: morphologically simple Dutch-German cognates, morphologically simple Dutch-German non-cognates, and morphologically complex opaque Dutch-German cognates. Cognates could be identical (e.g., verdienen (Dutch/German)) or non-identical (e.g., ondernemen (Dutch)/unternehmen (German)) translation equivalents whose common etymological origin was clear. Simple verbs had either a motor-related meaning or not; complex verbs contained either a motor-related stem or not, but had no motor-related meaning themselves (see Table 1 for the design). The selection of the materials used in the experiment was based on two types of online pretests: (a) a rating of the degree of motor-relatedness (scale from 1 'not motor-related' to 5 'highly motor-related') and an assessment of the body part involved, if any, of two pools of morphologically simple Dutch verbs (cognates and non-cognates); and (b) a rating of the transparency/opacity (scale from 1 'very opaque' to 5 'very transparent') of a pool of morphologically complex transparent opaque Dutch verb pairs, each pair sharing one of the above-mentioned simple cognate verbs as its stem. In the latter rating, participants were asked to rate the degree of semantic relatedness of the complex verbs and their stems. Transparent verbs were also included to present participants with the full range of transparency/opacity. Participants in each of these pretests (motor-relatedness: 20 participants each for simple cognate verbs and for simple non-cognate verbs; transparency/opacity: 39 participants) were Dutch L1 speakers of the same population as the fMRI experiment who did not participate in the other pretests nor in the fMRI experiment.

On the basis of these pretests, 48 pairs of cognate opaque complex verbs and their simple verb stems were selected, in addition to 48 non-cognate simple verbs. The simple verbs were either clearly motor-related or not, such that the simple motor verbs were judged to refer to movements to a higher degree than the simple non-motor verbs (see Appendix B in Supplementary Materials), as verified in $t$-tests (cognates: $t=12.08, p<.001$; non-cognates: $t=13.34, p<.001$ ). In addition, only simple motor verbs were selected that were judged to refer to hand movements by a majority of participants (cognates: $M=83.05 \%, \mathrm{SD}=17.33 \%$; non-cognates: $M=87.50 \%, \mathrm{SD}=14.22 \%$ ). The reasoning for this was that, if motor-related activations in (pre)motor areas are indeed somatotopically organized (Hauk et al., 2004 Raposo et al., 2009), selecting motor verbs associated with only one effector rather than a mixture of effectors would increase chances of detecting significant activations in (pre)motor brain areas. The complex cognate verbs selected on the basis of the pretests contained the simple cognate verbs as stems. In addition, they had been judged to be highly opaque, and the complex verbs with motor stems ('complex motor verbs') did not differ significantly from those with non-motor stems ('complex non-motor verbs') in terms of opacity ( $t=.70, p=.49)$. Within each category (simple cognate, simple non-cognate and complex verbs), motor and nonmotor verbs were also matched in terms of log-transformed lexical frequency ('log frequency', as determined by the Celex database, Baayen, Piepenbrock, \& Gulikers, 1995; all ps >.56) and length in letters (all ps $>.12$ ). See Appendix B in Supplementary Materials for an overview of stimulus characteristics.

The final set of experimental stimuli consisted of 96 Dutch morphologically simple verbs and 48 Dutch morphologically complex verbs. Half of the simple verbs
Table 2

Examples of stimuli.

\begin{tabular}{|c|c|c|}
\hline & Simple verb & Complex verb \\
\hline \multicolumn{3}{|l|}{ Cognate verb } \\
\hline Motor & nemen (nehmen/take) & $\begin{array}{l}\text { ondernemen } \\
\text { (unternehmen/undertake) }\end{array}$ \\
\hline Non-Motor & dienen (dienen/serve) & verdienen (verdienen/earn) \\
\hline \multicolumn{3}{|c|}{ Non-Cognate verb } \\
\hline Motor & gooien (werfen/throw) & \\
\hline Non-Motor & aarzelen (zögern/hesitate) & \\
\hline Pseudo-words & ralmen & verralmen \\
\hline
\end{tabular}

Note: German and English translations between parentheses.

(48) were Dutch-German cognates, while the other half were non-cognates. Within each simple verb category, half had a motor-related meaning, while the other half had not. All complex verbs were Dutch-German cognates and contained the simple cognate (motor or non-motor) verbs as stems, each preceded by a prefix (see Table 2 for examples). Finally, 24 pseudo-words were included. These were created by changing one or more letters of real Dutch words and obeyed the phonotactic rules of Dutch. All pseudo-words ended in the Dutch infinitive suffix 'en', as did the real words. Eight pseudo-words were morphologically complex, consisting of an existing prefix or particle followed by a non-existing stem. Sixteen pseudo-words were morphologically simple, consisting of a non-existing verb-like stem, 8 of which were also used as stem in the complex pseudo-words.

\subsection{Stimulus presentation}

Stimuli were projected on a mirror mounted on top of the head coil and read by participants lying on their back in the scanner. Participants held a response box in their right hand and responded only to pseudo-words by pressing a button with their right index finger. Each trial lasted for 8440 ms. First, a blank screen was presented for a variable jitter time $(0-2000 \mathrm{~ms})$, followed by a fixation cross, which remained in the center of the screen for $400 \mathrm{~ms}$. Then the stimulus word was presented for $2000 \mathrm{~ms}$ or until a response was given. Then a blank screen was presented until the trial duration of $8440 \mathrm{~ms}$ was reached.

The 144 experimental words and 24 pseudo-words were interspersed with 24 null events, consisting of a blank screen presented for $8440 \mathrm{~ms}$. The stimuli were pseudo-randomized such that no condition and no pseudo-words were presented on more than three consecutive trials. To exclude order effects, two pseudorandomizations were generated, each of which was also reversed, resulting in four lists. Participants were randomly assigned to one of the four lists. All stimuli were presented in one block, which lasted approximately $27 \mathrm{~min}$. Prior to the fMRI experiment, participants were familiarized with the task and test conditions in a practice session containing 8 trials, with words and pseudo-words not used in the experiment.

Following the fMRI-experiment, participants were presented with an off-line rating to find out whether they rated the motor versus non-motor verbs similarly as participants in the pretests. This rating contained the 144 experimental stimuli of the fMRI-experiment. Conditions were pseudo-randomized as described above. Participants were asked to judge the motor-relatedness of each of these verbs on a scale from 1 (not motor-related) to 5 (strongly motor-related).

Finally, L2 participants were asked to fill out the language background questionnaire summarized in Appendix A in Supplementary Materials.

\subsection{Behavioral data analysis}

Errors to words were analyzed for each language group separately, using two repeated-measures analyses of variance (ANOVAs) (see Table 1). The ANOVA for the Simple Verb subdesign, including only simple verbs, had Cognate Status (Cognate vs. Non-Cognate) and Motor-Relatedness (Motor vs. Non-Motor) as within-subject factors. The ANOVA for the Cognate Verb subdesign, including only cognate verbs, had Complexity (Simple vs. Complex) and Motor-Relatedness (Motor vs. NonMotor) as within-subject factors. Participants were excluded from the analysis if their percentage of errors to pseudo-words was higher than $40 \%$ and/or more than 2 standard deviations above the mean of their language group (L1 or L2 speakers, respectively). Items were excluded from the analysis if their percentage of errors was more than 3 standard deviations above the mean of their language group. Only correctly-answered trials were used in the fMRI analysis.

\section{5. fMRI data acquisition}

BOLD-sensitive functional images were acquired using a single-shot gradient EPI sequence ( 31 axial slices, $\mathrm{TR}=2110 \mathrm{~ms}, \mathrm{TE}=30 \mathrm{~ms}$, flip angle $=90^{\circ}$, voxel size $=3.5 \times 3.5 \times 3.5 \mathrm{~mm}^{3}$ ). After acquisition of the EPI images, high-resolution 
anatomical images were acquired using an MPRAGE sequence (192 sagittal slices, $\mathrm{TR}=2300 \mathrm{~ms}, \mathrm{TE}=3.03 \mathrm{~ms}, \mathrm{FOV}=256$, voxel size $=1 \times 1 \times 1 \mathrm{~mm}^{3}$ ). All images were acquired on a Siemens TRIO 3.0 T MRI System (Siemens, Erlangen, Germany), using a birdcage head coil for radio-frequency transmission and signal reception.

\section{6. fMRI data analysis}

Data analysis was conducted with SPM8 (Statistical Parametric Mapping, www. fil.ion.ucl.ac.uk/spm). The first five volumes were discarded to allow for T1 equilibration effects. Then, functional images were corrected for motion artifacts through rigid body registration along three translations and three rotations. The realigned images were corrected for differences in acquisition time using the middle slice (slice 17) as reference. The resulting images were normalized to the standard stereotactic Montreal Neurological Institute (MNI) coordinate system. For this, the mean functional image was co-registered with the T1-image using a rigidbody model. Then, spatial-normalization parameters were generated, which were used to normalize the functional images into standard MNI space using trilinear interpolation, while resampling the images at an isotropic voxel size of $2 \mathrm{~mm}$. For one L2 participant, this normalization method led to considerable distortion. Therefore, this participant's images were normalized to a standard EPI template centered in MNI space, likewise resampling them at an isotropic voxel size of $2 \mathrm{~mm}$. Finally, the normalized images were smoothed with an isotropic 8-mm FWHM Gaussian kernel. The resulting preprocessed images were analyzed on a subject-bysubject basis using the general linear model, with regressors entered for each critical condition (Simple/Cognate/Motor, Simple/Cognate/Non-Motor, Simple/NonCognate/Motor, Simple/Non-Cognate/Non-Motor, Complex/Motor, Complex/NonMotor) as well as null trials and convolved with a canonical hemodynamic response function. The realignment parameters calculated during motion correction were included in the model as effects of no interest.

\subsubsection{ROI analyses}

We conducted ROI analyses for (pre)motor and somatosensory areas to test whether L1 and L2 speakers were sensitive to the motor-related meaning of simple verbs and of complex verb stems. To create these ROIs, we selected the peaks of activation reported by Rüschemeyer et al. (2007) for the interaction between Complexity and Motor-Relatedness in the left postcentral gyrus and the left parietal operculum (S2). However, since the postcentral gyrus peak reported by Rüschemeyer et al. (2007) is slightly more posterior than what is usually reported for motor words (Hauk et al., 2004; Postle et al., 2008; Willems, Toni, Hagoort, \& Casasanto, 2009), we also created a more anterior ROI. As our study was a partial replication of Rüschemeyer et al.'s study, we also based this ROI on peaks of activation reported by the former study, but this time for the Simple Motor versus Non-Motor contrast. Two (pre)motor peaks of activation were reported for this contrast, i.e. [ $-44-15$ 59] (central sulcus) and [ $-47-9$ 57] (precentral gyrus), of which we calculated the average. The resulting coordinates for the three ROIs were then converted from Talairach to MNI stereotactic space. In this way, the [ $-38-2156]$ postcentral gyrus, the [ $-47-1514]$ S2 and the [ $-46-1258]$ (pre)motor coordinates were converted to [ $-38-2560],[-47-1614]$ and [-46 - 17 64], respectively. Using Marsbar (Brett, Anton, Valabregue, \& Poline, 2002), ROIs were created as a sphere with a $10 \mathrm{~mm}$ radius with each of the selected coordinates as a center. Since the ROI with the postcentral peak extended from the post- to the precentral gyrus, including both somatosensory and motor areas, this ROI was dubbed the sensorimotor (SM) ROI. The ROI with the S2 peak was named the S2 ROI and the ROI with the peak in the motor/premotor area was called the Pre/M ROI (see Fig. 1).

For all these ROIs, contrast values were obtained for each condition (compared to the null condition) for each participant. Next, repeated-measures analyses of variance (ANOVAs) were performed on these contrast values. For each subdesign, an omnibus repeated-measures ANOVA including both language groups was performed. For the Simple Verb subdesign, the ANOVA had the factors of Cognate Status (Cognate vs. NonCognate), Motor-Relatedness (Motor vs. Non-Motor) and Language (L1 vs. L2). For the Cognate Verb subdesign, the ANOVA had the factors of Complexity (Simple vs. Complex), Motor-Relatedness (Motor vs. Non-Motor) and Language (L1 vs. L2). In all ANOVAs, a significance level of $\alpha=.05$ was used. Only main effects and interactions involving Motor-Relatedness are discussed. Follow-up analyses were performed only to significant or marginally significant interactions involving Motor-Relatedness.

\subsubsection{Whole-brain analyses}

To further investigate effects at the whole-brain level, six contrast images were generated for each participant: the baseline-corrected effects of simple cognate motor and non-motor verbs, of simple non-cognate motor and non-motor verbs and of complex motor and non-motor verbs (i.e. each experimental condition compared to the null condition). Again, analyses were done for the two subdesigns separately. For the Simple Verb subdesign, the four contrast images for simple verbs of all participants were entered into a full-factorial $(2 \times 2 \times 2)$ second-level random effects analysis over both language groups. The three factors specified were Cognate Status, Motor-Relatedness and Language. To further investigate the nature of representations of simple motor versus non-motor verbs, directional $t$-tests were performed for the main effect of Motor-Relatedness (i.e. [Simple (Motor-NonMotor)] and the reverse contrast). In addition, the interactions involving Language

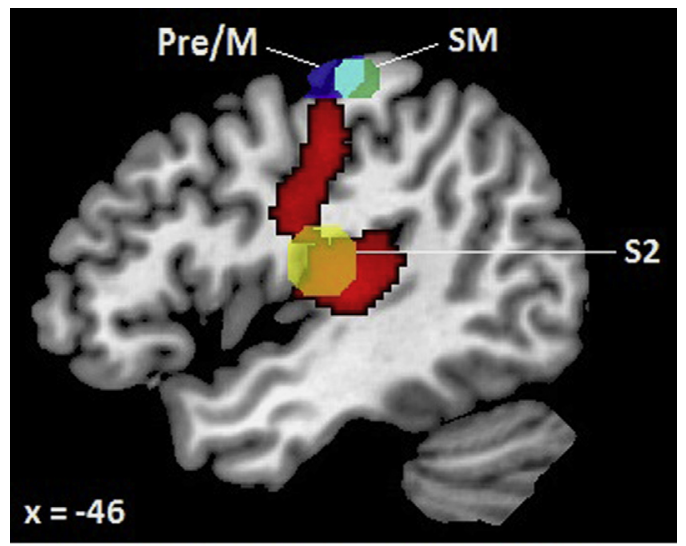

Simple (Motor - Non-Motor) [Simple Verbs] Overlap SM \& Pre/M ROIs $\square$ Overlap Pre/M ROI \& Simple [Motor - Non-Motor]

Fig. 1. Overlap between full-factorial [Simple (Motor-Non-Motor)] contrast (Simple Verb subdesign) over both groups (red) and S2 ROI (yellow), SM ROI (green), and Pre/M ROI (blue). (For interpretation of the references to color in this figure legend, the reader is referred to the web version of this article.)

and Motor-Relatedness were tested to investigate possible differences between L1 and L2 speakers. For this, we again used directional $t$-contrasts, with equal weights attributed to each condition (e.g., [1 $11-1-1-1-111]$ for the (L1 (Motor-NonMotor))-(L2 (Motor-Non-Motor)) interaction contrast). To study the two language groups in more detail, full-factorial $(2 \times 2)$ analyses were performed for each language group separately. Each of these second-level random effects analyses contained Cognate Status and Motor-Relatedness as factors, and t-tests were performed for the main effect of Motor-Relatedness and the interaction between Cognate Status and Motor-Relatedness.

For the Cognate Verb subdesign, the four contrast images of all participants for cognate verbs were entered into another full-factorial $(2 \times 2 \times 2)$ second-level random effects analysis over both language groups. The three factors specified in this design were Complexity, Motor-Relatedness and Language. The nature of representations of simple versus complex motor and non-motor verbs was further investigated with directional $t$-tests performed for the interaction between Complexity and MotorRelatedness (i.e. [(Simple Cognate (Motor-Non-Motor))-(Complex (Motor-Non-

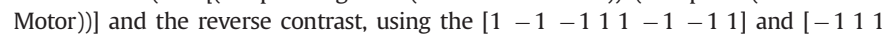
$-1-1111-1$ ] contrast vectors, respectively). Significant activations found for this contrast led to follow-up analyses on the Motor-Relatedness effect of simple cognate verbs (i.e. [Simple Cognate (Motor-Non-Motor)] and the reverse contrast) and of complex verbs (i.e. [Complex (Motor-Non-Motor)] and the reverse contrast). In addition, the triple interaction between Complexity, Motor-Relatedness and Language was tested with directional $t$-contrasts to study possible differences between language groups. Again, the two language groups were then investigated in separate fullfactorial $(2 \times 2)$ analyses, containing Complexity and Motor-Relatedness as factors. $T$-contrasts were tested for the interaction between Complexity and Motor-Relatedness and the effect of Motor-Relatedness for simple cognate and complex verbs separately.

A double threshold was used to protect against false-positive activations, by combining a voxel-level $p$-value of $p<.001$ (uncorrected) with a minimum cluster extent of 43 voxels $(p<.001 / k=43$ threshold). This cluster extent was determined by modeling the entire imaging volume, assuming a specified individual voxel type error (in this case $p=.001$ ), smoothing the volume with a three-dimensional 8-mm FWHM Gaussian kernel and calculating the probability associated with each cluster extent across 1000 Monte Carlo simulations, yielding a correction for multiple comparisons of $p<.05$ (Forman et al., 1995; Slotnick, Moo, Segal, \& Hart Jr., 2003). When the predicted activations in sensorimotor areas were not found with the $p<.001 / k=43$ threshold, an alternative threshold was used. For this, a voxel-level $p$ value of $p<.005$ (uncorrected) was combined with a minimum cluster extent of 65 voxels ( $p<.005 / k=65$ threshold), also leading to a correction for multiple comparisons of $p<.05$ according to the method described above (with the increased cluster size compensating for the higher threshold). The $p<.005 / k=65$ threshold was used to make sure that the lack of a predicted activation was not due to a thresholding issue. When the $p<.005 / k=65$ threshold was used for a certain contrast, then it was also used for its reverse contrast, to take into account related thresholding issues. The same was done for related simple contrasts yielding no significant activations in sensorimotor areas with the $p<.001 / k=43$ threshold.

\section{Results}

Of the 51 participants, seven (one L1 speaker, six L2 speakers) were excluded because their percentage of errors to pseudo-words 
was higher than $40 \%$ and/or more than 2 standard deviations above the mean. In addition, six participants (one L1 speakers, five L2 speakers) were excluded because of compromised data quality, technical problems and/or motion artifacts. For the L1 group, three items were excluded because their percentage of errors was more than 3 standard deviations above the mean. This was also the case for two items for the L2 group.

\subsection{Behavioral results}

Table 3 provides an overview of the errors to words. L1 speakers made relatively few errors to word or pseudo-word stimuli $(M=1.54 \%, \mathrm{SD}=3.10 \%$, and $M=5.94 \%, \mathrm{SD}=8.60 \%$, respectively), indicating that they were attentive to the task. The analysis of their errors to simple verbs only (Simple Verb subdesign) revealed no significant effects or interactions ( $F s<2.39$, ps $>.13$ ). The ANOVA for the Cognate Verb subdesign showed a significant interaction between Complexity and Motor-Relatedness $(F(1,19)=$ $4.61, p<.05)$, due to a tendency to make more errors to complex motor compared to non-motor verbs $(t=2.03, p=.057)$. Simple cognate motor and non-motor verbs did not differ $(p>.57)$. The main effects of Motor-Relatedness and Complexity were not significant $(F s<1.74, p s>.20)$.

L2 speakers made somewhat more errors to word and pseudoword stimuli than L1 speakers $(M=9.79 \%, \mathrm{SD}=9.36 \%$, and $M=12.67 \%, \mathrm{SD}=12.18 \%$, respectively). The analysis of their errors to simple verbs only (Simple Verb subdesign) revealed a significant main effect of Cognate Status $(F(1,17)=11.70, p<.01)$, indicating that L2 speakers made more errors to non-cognate than to cognate simple verbs. Other effects and interactions did not reach significance $(F s<.46, p s>.50)$. The ANOVA for the Cognate Verb subdesign showed a marginally significant main effect of Complexity, indicating that there were somewhat more errors to complex than to simple cognate verbs $(F(1,17)=3.10, p=.096)$. Other effects and interactions were not significant $(F \mathrm{~s}<1.78$, $p s>.20)$. See Table 3 for an overview.

\subsection{Off-line rating results}

The results of the off-line motor-relatedness rating conducted after the fMRI-experiment showed that both L1 and L2 participants judged simple cognate motor verbs (L1: $M=4.11, \mathrm{SD}=.54$; L2: $M=4.09, \mathrm{SD}=.42$ ) and simple non-cognate motor verbs (L1: $M=4.50, \mathrm{SD}=.33 ; \mathrm{L} 2: M=4.29, \mathrm{SD}=.49$ ) to be significantly more motor-related than simple cognate non-motor verbs ( $\mathrm{L} 1: \mathrm{M}=2.21$, $\mathrm{SD}=.56 ; \mathrm{L} 2: M=1.74, \mathrm{SD}=.37)$ and simple non-cognate non-motor verbs ( $\mathrm{L} 1: M=1.81, \mathrm{SD}=.45 ; \mathrm{L} 2: M=1.82, \mathrm{SD}=.41)$, respectively (L1: ts $>15.67, p s<.001$; L2: ts $>14.78, p s<.001$ ). The difference between complex motor verbs ( $\mathrm{L} 1: M=2.24, \mathrm{SD}=.44$; $\mathrm{L} 2: M=2.24$, $\mathrm{SD}=.52)$ and complex non-motor verbs (L1: $M=1.98, \mathrm{SD}=.47 ; \mathrm{L} 2$ :

Table 3

Behavioral results: \% Errors to words.

\begin{tabular}{lcc}
\hline & L1 speakers & L2 speakers \\
\hline Simple Cognate & & \\
Motor & $1.04(1.85)$ & $5.79(5.37)$ \\
Non-Motor & $1.46(2.80)$ & $7.00(5.99)$ \\
Simple Non-Cognate & & \\
Motor & $.42(1.28)$ & $13.19(12.56)$ \\
Non-Motor & $1.52(2.55)$ & $13.89(11.96)$ \\
Complex Cognate & & $10.42(7.32)$ \\
Motor & $3.26(5.06)$ & $8.45(8.63)$ \\
Non-Motor & $1.52(3.24)$ & \\
\hline
\end{tabular}

Note: Mean percentages shown, with standard deviations between parentheses.
$M=1.67, \mathrm{SD}=.54)$ was small but significant (L1: $t=3.92, p<.001$; L2: $t=9.54, p<.001)$.

\section{3. fMRI results: Simple Verb subdesign}

\subsubsection{ROI analyses}

To investigate whether simple motor verbs elicited more activation in somatosensory and motor areas than simple nonmotor verbs, three ROIs were tested: the S2, the SM and the Pre/M ROI. For each ROI, an omnibus ANOVA over both language groups was followed up by ANOVAs for each group separately (see Fig. 2 for an overview of results).

3.3.1.1. S2 ROI. For the S2 ROI, the Simple Verb omnibus ANOVA including both language groups (see Table 4) showed that simple motor verbs yielded significantly higher activation in the S2 ROI than simple non-motor verbs, as indicated by a main effect of Motor-Relatedness. The triple interaction between Cognate Status, Motor-Relatedness and Language was marginally significant. No other effects or interactions involving Motor-Relatedness were significant.

In the analyses for L1 and L2 speakers separately, the interaction between Cognate Status and Motor-Relatedness did not reach significance. In contrast, the main effect of Motor-Relatedness was significant for both groups. These results indicate that simple motor verbs elicited greater levels of activation in the S2 ROI than simple non-motor verbs in both language groups, regardless of cognate status.

3.3.1.2. SM and Pre/M ROIs. For the other two ROIs, the Simple Verb omnibus ANOVA including both language groups (SM ROI: Table 5; Pre/M ROI: Table 6) showed a similar pattern of results. A significant main effect of Motor-Relatedness indicated that there was more activation for simple motor than non-motor verbs in these ROIs. No other effects or interactions involving Motor-Relatedness were significant.

For the SM ROI, separate analyses for the two language groups indicated that the same pattern could be found with both groups: Both L1 and L2 speakers showed a main effect of Motor-Relatedness, whereas the interaction between Cognate Status and MotorRelatedness was not significant in either group.

For the Pre/M ROI, separate analyses for both language groups revealed that the main effect of Motor-Relatedness was significant with L1 speakers, but not L2 speakers. No other effects or interactions involving Motor-Relatedness were found with either language group.

Thus, motor-related activations were found with both language groups in the SM ROI and with L1 speakers in the Pre/M ROI.

\subsubsection{Whole-brain analysis}

To find out whether simple motor verbs elicited more activation than simple non-motor verbs in other brain regions, fullfactorial whole-brain analyses were done over both language groups together and for each group separately.

\subsubsection{Analysis over both groups}

3.3.2.1.1. Simple (Motor-Non-Motor)and reverse contrast. In the Simple Verb analysis over both groups, the [Simple (Motor-NonMotor)] contrast revealed significant clusters of activation in somatosensory and motor areas (see Fig. 3). Bilaterally, a large cluster extended from the superior and transverse temporal gyri over the parietal and rolandic operculum, the insula, the claustrum and the putamen to the pre- and postcentral gyri and central sulcus. Activations were also found from the bilateral midcingulate gyrus over the medial frontal gyrus into the right superior frontal gyrus and 
L1

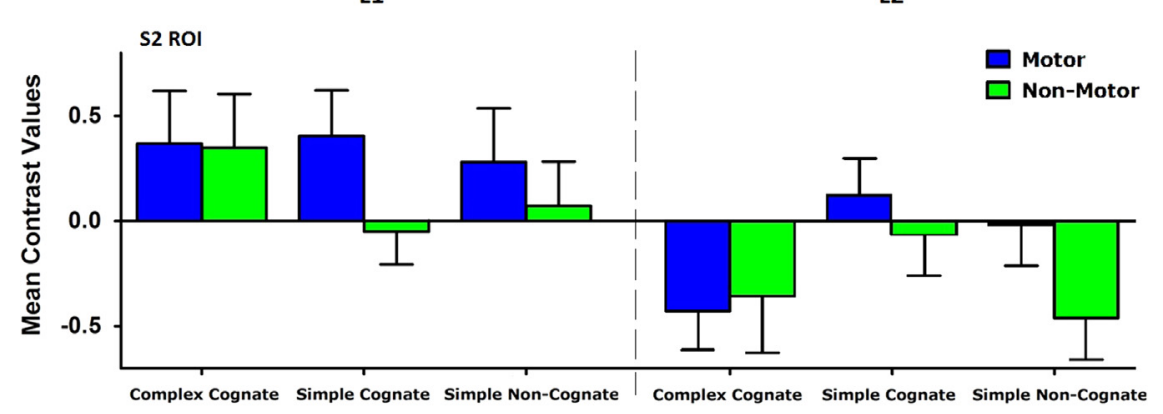

Complexity \& Cognate Status
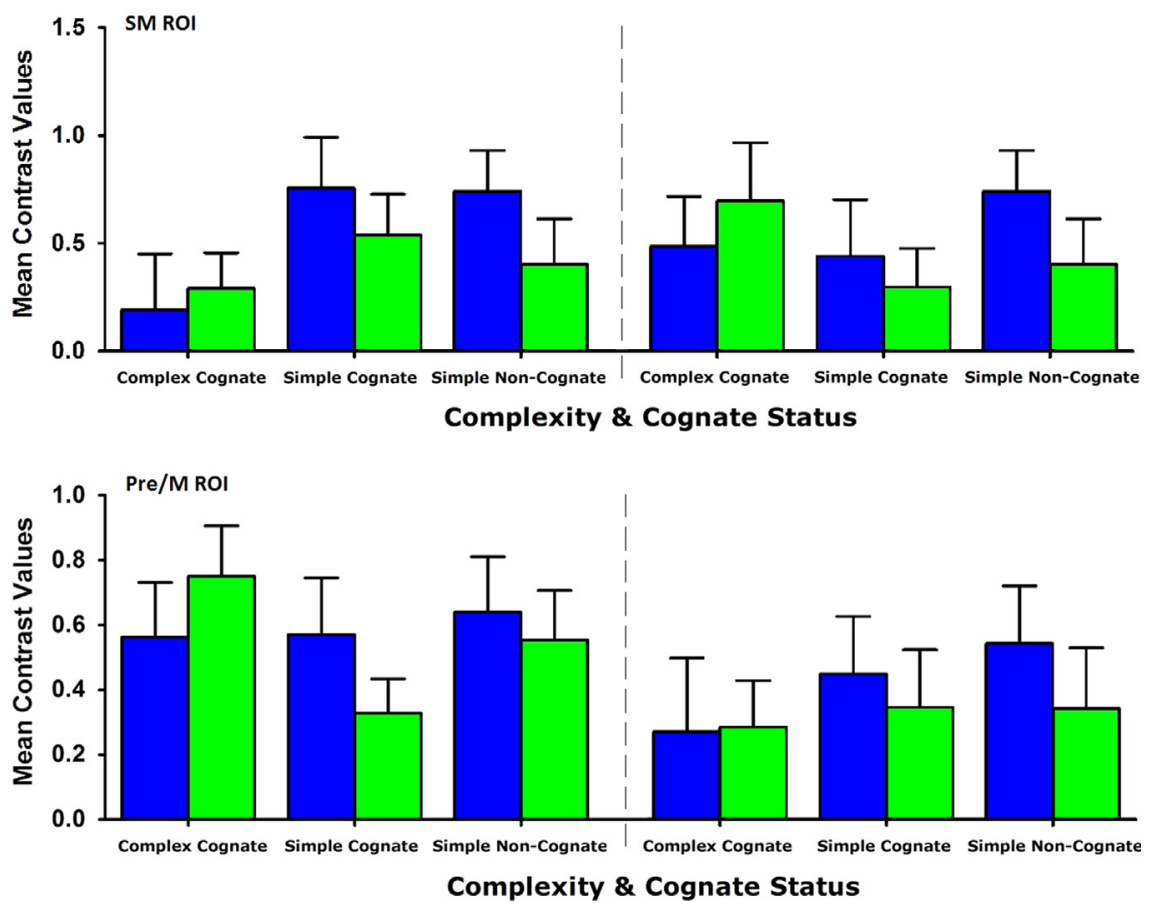

Fig. 2. ROI analyses: Contrast values for each of the experimental conditions for L1 and L2 speakers (error bars: $+1 \mathrm{SE}$ ).

Table 4

S2 ROI: Simple Verb ANOVA on contrast values for simple verbs for L1 and L2 speakers.

\begin{tabular}{|c|c|c|c|c|c|c|}
\hline \multirow[t]{2}{*}{ Effect } & \multicolumn{2}{|c|}{ Both groups } & \multicolumn{2}{|c|}{ L1 speakers } & \multicolumn{2}{|c|}{ L2 speakers } \\
\hline & F-value & p-value & F-value & p-value & F-value & $p$-value \\
\hline Cognate Status & 2.68 & .11 & $<.001$ & .99 & 4.94 & .04 \\
\hline Motor & 15.52 & $<.001$ & 6.48 & .02 & 11.13 & .004 \\
\hline Cognate Status $\times$ Motor & .002 & .97 & 1.55 & .23 & 1.96 & .18 \\
\hline Language & 1.22 & .28 & NA & NA & NA & NA \\
\hline Cognate Status $\times$ Language & 2.64 & .11 & NA & NA & NA & NA \\
\hline Motor $\times$ Language & .01 & .92 & NA & NA & NA & NA \\
\hline Cognate Status $\times$ Motor $\times$ Language & 3.43 & .07 & NA & NA & NA & NA \\
\hline
\end{tabular}

Notes: df: Both groups: 1,36; L1 speakers: 1,19; L2 speakers: 1, 17. Motor: Motor-Relatedness. NA: Not applicable.

paracentral lobule. Ventrally, activations were seen in the bilateral amygdala and hippocampus. More caudally, clusters were found stretching from the right anterior lingual gyrus to the right calcarine fissure and from the left parieto-occipital to the calcarine fissure.

The reverse contrast yielded two significant clusters in the left inferior frontal gyrus, one in the pars orbitalis, one in the pars triangularis.

3.3.2.1.2. Interactions involving Motor-Relatedness and Language. The interaction between Motor-Relatedness and Language [i.e. (L1 (Simple Motor-Non-Motor))-(L2 (Simple Motor-Non-Motor))] revealed a significant cluster in the left supramarginal gyrus, and one in the right anterior calcarine fissure. For the reverse contrast, no significant clusters were found.

The three-way interaction between Cognate Status, MotorRelatedness and Language [i.e. (L1 (Simple Cognate (Motor-NonMotor)-Simple Non-Cognate (Motor-Non-Motor)))-(L2 (Simple Cognate (Motor-Non-Motor)-Simple Non-Cognate (Motor-NonMotor)))] revealed a significant cluster in the right claustrum and putamen, whereas no significant clusters were found with the reverse contrast. 
Table 5

SM ROI: Simple Verb ANOVA on contrast values for simple verbs for L1 and L2 speakers.

\begin{tabular}{|c|c|c|c|c|c|c|}
\hline \multirow[t]{2}{*}{ Effect } & \multicolumn{2}{|c|}{ Both groups } & \multicolumn{2}{|c|}{ L1 speakers } & \multicolumn{2}{|c|}{ L2 speakers } \\
\hline & F-value & p-value & F-value & p-value & F-value & p-value \\
\hline Cognate Status & .22 & .64 & 1.38 & .26 & .57 & .46 \\
\hline Motor & 9.74 & .004 & 4.27 & .053 & 5.31 & .034 \\
\hline Cognate Status $\times$ Motor & .58 & .45 & .24 & .63 & .34 & .57 \\
\hline Language & .33 & .57 & NA & NA & NA & NA \\
\hline Cognate Status $\times$ Language & 1.87 & .18 & NA & NA & NA & NA \\
\hline Motor $\times$ Language & .32 & .57 & NA & NA & NA & NA \\
\hline Cognate Status $\times$ Motor $\times$ Language & .006 & .94 & NA & NA & NA & NA \\
\hline
\end{tabular}

Notes: df: Both groups: 1,36; L1 speakers: 1,19; L2 speakers: 1,17. Motor: Motor-Relatedness and NA: Not applicable.

Table 6

Pre/M ROI: Simple Verb ANOVA on contrast values for simple verbs for L1 and L2 Speakers.

\begin{tabular}{|c|c|c|c|c|c|c|}
\hline \multirow[t]{2}{*}{ Effect } & \multicolumn{2}{|c|}{ Both groups } & \multicolumn{2}{|c|}{ L1 speakers } & \multicolumn{2}{|c|}{ L2 speakers } \\
\hline & F-value & p-value & F-value & p-value & F-value & p-value \\
\hline Cognate Status & 3.58 & .066 & 4.42 & .049 & .38 & .55 \\
\hline Motor & 8.82 & .005 & 7.57 & .013 & 2.82 & .11 \\
\hline Cognate Status $\times$ Motor & .12 & .74 & 1.44 & .25 & .83 & .38 \\
\hline Language & .22 & .65 & NA & NA & NA & NA \\
\hline Cognate Status $\times$ Language & .99 & .33 & NA & NA & NA & NA \\
\hline Motor $\times$ Language & .01 & .91 & NA & NA & NA & NA \\
\hline Cognate Status $\times$ Motor $\times$ Language & 2.21 & .15 & NA & NA & NA & NA \\
\hline
\end{tabular}

Notes: df: Both groups: 1,36; L1 speakers: 1,19; L2 speakers: 1,17. Motor: Motor-Relatedness and NA: Not applicable.

None of the clusters found for the interactions involving MotorRelatedness and Language overlapped with the clusters found for the [Simple (Motor-Non-Motor)] contrast.

\subsubsection{Analysis of the L1 group}

3.3.2.2.1. Simple (Motor-Non-Motor) and reverse contrast. In the Simple Verb analysis of L1 speakers only, the [Simple (Motor-NonMotor)] contrast yielded a similar pattern as the analysis over both groups (Fig. 4). Bilaterally, significant clusters extended from the postcentral gyrus to the central sulcus and to the right precentral gyrus, and from the parietal and rolandic operculum to the transverse temporal gyrus. More caudally, significant clusters were seen in the right anterior lingual gyrus and in the left parietooccipital fissure. Other significant clusters were found in the left supramarginal gyrus, in the left amygdala and in the left putamen reaching into the globus pallidus.

The reverse contrast yielded no significant clusters.

3.3.2.2.2. Interaction between Cognate Status and MotorRelatedness. The [(Simple Cognate (Motor - Non-Motor))-(Simple Non-Cognate (Motor-Non-Motor))] contrast and its reverse did not reveal any significant clusters.

\subsubsection{Analysis of the L2 group}

3.3.2.3.1. Simple (Motor-Non-Motor) and reverse contrast. When L2 speakers were analyzed separately, the [Simple (Motor-NonMotor)] contrast also revealed significant activation in somatosensory and motor areas (Fig. 4). Clusters extended from the bilateral transverse temporal gyrus to the bilateral parietal operculum. In the right hemisphere, another significant cluster stretched from the precentral gyrus through the central sulcus to the postcentral gyrus. Subcortically, activations were found in the bilateral amygdala. All these clusters, except the one in the right amygdala, overlapped with the clusters found for the same contrast in the analysis including L1 speakers only.
The reverse contrast only revealed a significant cluster on the border of the pars triangularis and the pars orbitalis of the left inferior frontal gyrus.

3.3.2.3.2. Interaction between Cognate Status and MotorRelatedness. This interaction yielded significant clusters of activation in both directions in regions associated with executive control. The [(Simple Cognate (Motor-Non-Motor))-(Simple Non-Cognate (MotorNon-Motor))] contrast showed a left-hemisphere cluster extending from the caudal anterior cingulate cortex to the medial and superior frontal gyrus. Another left-hemisphere cluster was found in the inferior precentral sulcus. In the right hemisphere, significant clusters were found in the anterior insula and in the corpus callosum.

The reverse contrast revealed two clusters: one stretching from the ventral anterior cingulate gyrus to the anterior medial frontal gyrus bilaterally (but more extensively in the right hemisphere), and another cluster in the right angular gyrus.

The clusters found for the interactions with Motor-Relatedness did not overlap with the clusters found for the [Simple (MotorNon-Motor)] contrast. To rule out the possibility that the somatosensory and motor activations found for the [Simple (Motor-NonMotor)] contrast (see above) were driven by the simple cognate verbs only, we ran an additional analysis contrasting simple non-cognate motor and non-motor verbs. The results show that somatosensory and motor regions are not only activated when we look at cognate and non-cognate verbs together, but also when only non-cognate verbs are included in the analysis (for more detailed results, see Appendix C in Supplementary Materials).

3.3.2.4. Summary. To summarize, the [Simple (Motor-Non-Motor)] contrast yielded significant activations in motor and somatosensory areas (i.e. pre- and postcentral gyri, central sulcus and parietal operculum), both in the analysis over the two language groups and in the analyses for each language group separately. For L1 speakers, the interaction between Cognate Status and Motor-Relatedness revealed no significant activations, 


\section{Both groups}

a

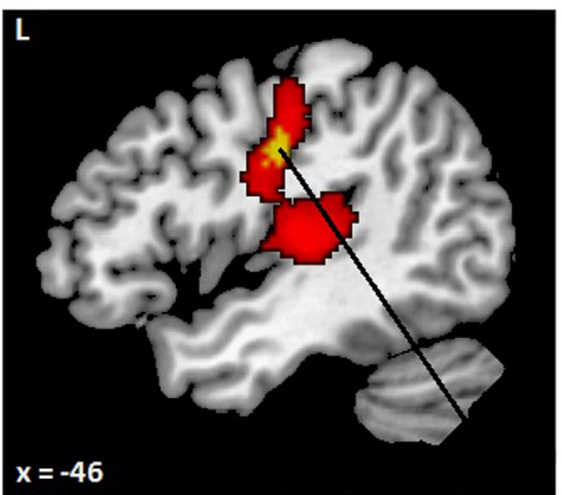

b

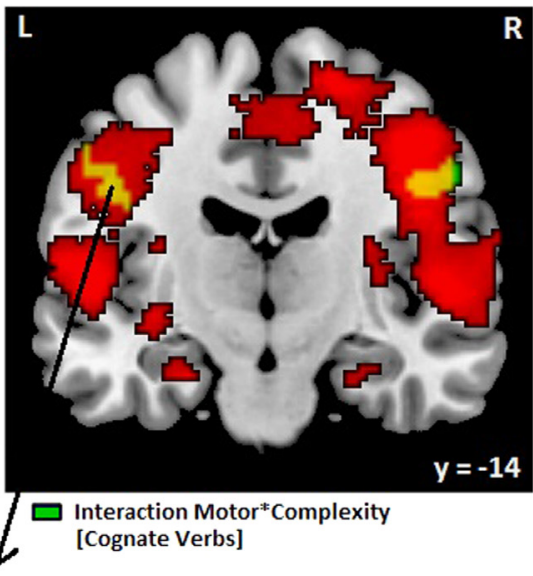

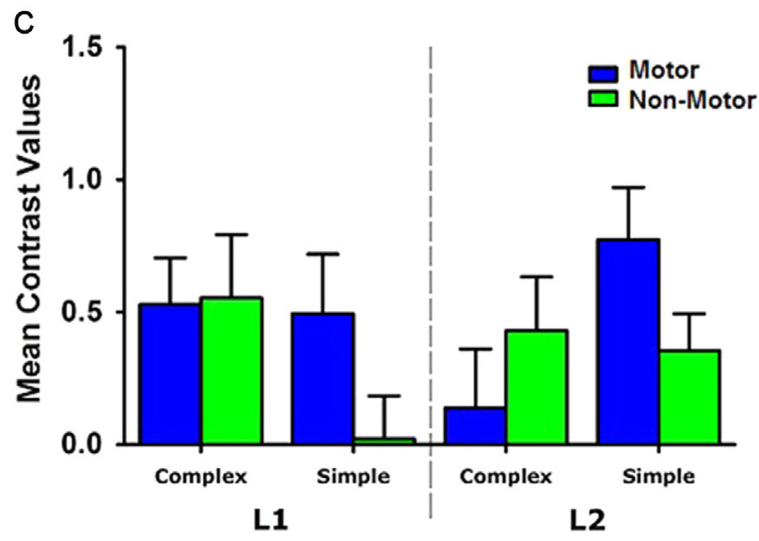

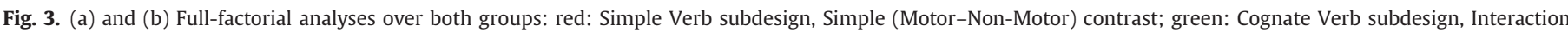

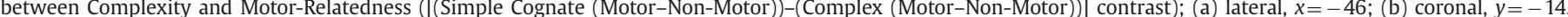

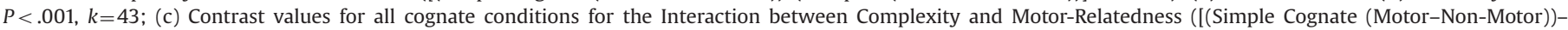

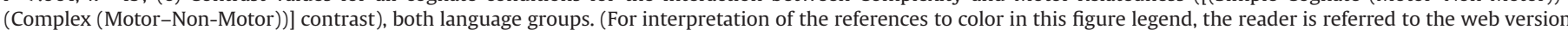
of this article.)

\section{Simple Verbs: Simple (Motor - Non-Motor)}

a

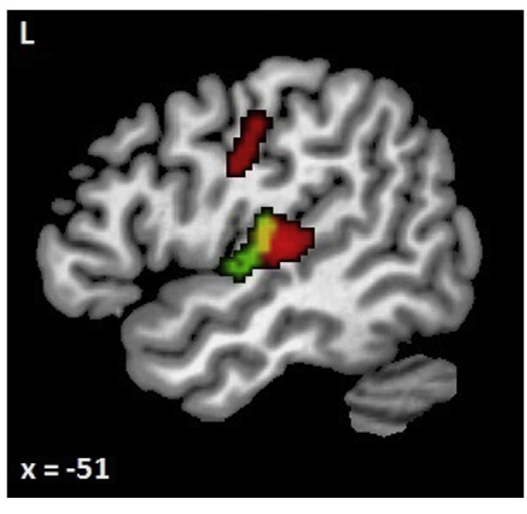

\section{b}

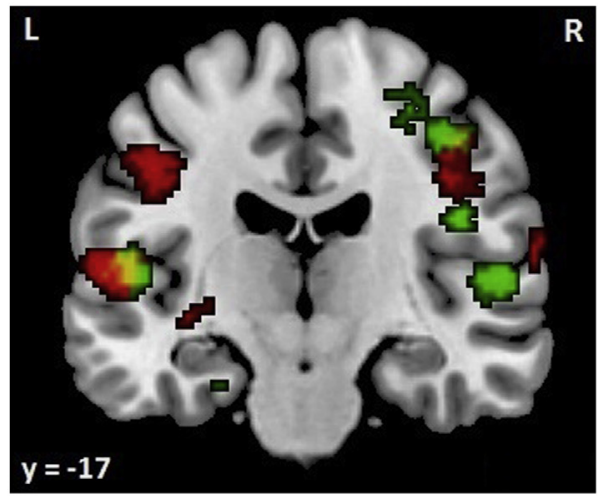

L2 $\square$ overlap

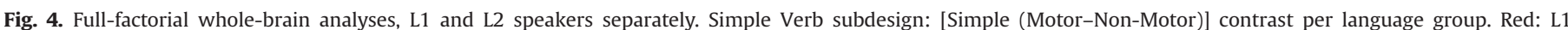

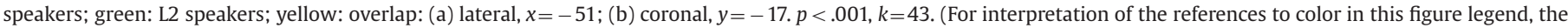
reader is referred to the web version of this article.)

whereas L2 speakers showed clusters of significant activation for this interaction (in both directions) in areas associated with executive control and decision processes (anterior cingulate cortex, medial frontal gyrus and inferior precentral sulcus; see Appendix D in Supplementary Materials for a list of significant activations for the [Simple (Motor-Non-Motor)] contrast). 
Table 7

S2 ROI: Cognate Verb ANOVA on contrast values for cognate verbs for L1 and L2 speakers.

\begin{tabular}{|c|c|c|c|c|c|c|}
\hline \multirow[t]{2}{*}{ Effect } & \multicolumn{2}{|c|}{ Both groups } & \multicolumn{2}{|c|}{ L1 speakers } & \multicolumn{2}{|c|}{ L2 speakers } \\
\hline & F-value & p-value & F-value & p-value & F-value & p-value \\
\hline Complexity & 1.76 & .19 & 1.48 & .24 & 20.59 & $<.001$ \\
\hline Motor & 3.25 & .08 & 4.39 & .05 & .24 & .63 \\
\hline Complexity $\times$ Motor & 4.59 & .039 & 3.44 & .079 & 1.38 & .26 \\
\hline Language & 2.79 & .104 & NA & NA & NA & NA \\
\hline Complexity $\times$ Language & 11.16 & .002 & NA & NA & NA & NA \\
\hline Motor $\times$ Language & 1.20 & .28 & NA & NA & NA & NA \\
\hline Complexity $\times$ Motor $\times$ Language & .31 & .58 & NA & NA & NA & NA \\
\hline
\end{tabular}

Notes: df: Both groups: 1,36; L1 speakers: 1,19; L2 speakers: 1,17. Motor: Motor-Relatedness . NA: Not applicable.

Table 8

SM ROI: Cognate Verb ANOVA on contrast values for cognate verbs for L1 and L2 Speakers.

\begin{tabular}{|c|c|c|c|c|c|c|}
\hline \multirow[t]{2}{*}{ Effect } & \multicolumn{2}{|c|}{ Both groups } & \multicolumn{2}{|c|}{ L1 speakers } & \multicolumn{2}{|c|}{ L2 speakers } \\
\hline & F-value & $p$-value & F-value & p-value & F-value & p-value \\
\hline Complexity & 1.15 & .29 & 2.53 & .13 & 18.58 & $<.001$ \\
\hline Motor & .02 & .88 & .14 & .71 & .17 & .69 \\
\hline Complexity $\times$ Motor & 3.02 & .091 & 1.76 & .20 & 1.29 & .27 \\
\hline Language & .02 & .90 & NA & NA & NA & NA \\
\hline Complexity $\times$ Language & 13.32 & .001 & NA & NA & NA & NA \\
\hline Motor $\times$ Language & .31 & .58 & NA & NA & NA & NA \\
\hline Complexity $\times$ Motor $\times$ Language & .008 & .93 & NA & NA & NA & NA \\
\hline
\end{tabular}

Notes: df: Both Groups: 1,36; L1 speakers: 1,19; L2 speakers: 1,17. Motor: Motor-Relatedness. NA: Not applicable.

\section{4. fMRI results: Cognate Verb subdesign}

\subsubsection{ROI analyses}

To find out whether both language groups showed motorrelated activations for simple cognate but not complex cognate verbs, the S2, SM and Pre/M ROIs were tested with ANOVAs over both language groups and for each group separately (see Fig. 2 for an overview of the results)

3.4.1.1. S2 ROI. In the Cognate Verb omnibus ANOVA including both language groups, the S2 ROI (Table 7) showed a marginally significant effect of Motor-Relatedness, modulated by a significant interaction between Complexity and Motor-Relatedness. Planned pair-wise comparisons indicated that simple cognate motor verbs elicited more activation in the S2 ROI than simple cognate nonmotor verbs $(t=2.94, p<.01)$, whereas complex motor and nonmotor verbs did not differ $(t=-.20, p=.84)$. These results suggest that motor-related activations are only seen with simple verbs and not with opaque complex verbs, replicating Rüschemeyer et al.'s (2007) findings.

When L1 speakers were analyzed separately, a similar pattern was found: A significant main effect of Motor-Relatedness was modulated by a marginally significant interaction between Complexity and Motor-Relatedness. Follow-up comparisons again indicated that simple cognate motor verbs induced significantly greater levels of activation in the S2 ROI than simple cognate non-motor verbs $(t=2.57, p<.01)$, while complex motor and nonmotor verbs did not differ $(t=.12, p>.45){ }^{2}$

When L2 speakers were analyzed separately, the main effect of Motor-Relatedness and its interaction with Complexity did not

\footnotetext{
${ }^{2}$ Note that a direct comparison of the activation levels for simple versus complex verbs cannot be interpreted in a straightforward manner, as simple and complex verbs were not matched on a number of parameters. We will come back to this issue in Section 4.
}

reach significance. This lack of significance with L2 speakers is discussed in more detail in Section 4.

3.4.1.2. SM and Pre/M ROIs. In the Cognate Verb omnibus ANOVAs, the SM and Pre/M ROIs displayed similar patterns as the S2 ROI: The interaction between Complexity and Motor-Relatedness was significant for the Pre/M ROI (Table 9), and marginally significant for the SM ROI (Table 8). As confirmed by planned pair-wise comparisons, simple cognate motor verbs elicited more activation in the Pre/M ROI $(t=2.38, p<.05)$ and the SM ROI $(t=1.60$, $p=.059)$ than simple cognate non-motor verbs, whereas the difference between complex motor and non-motor verbs did not come close to significance ( $t \mathrm{~s}<1.20, p \mathrm{~s}>.24$ ). No other significant effects or interactions involving Motor-Relatedness were found in these analyses. Again, Rüschemeyer et al.'s findings seem to be replicated for the Pre/M and the SM ROI, although less robustly for the SM ROI.

The less robust nature of the replication for the SM ROI is also illustrated by the separate analyses of the two language groups: No significant effects or interactions involving Motor-Relatedness were found in either group. For the Pre/M ROI, on the other hand, the replication was more robust: For L1 speakers, the interaction between Complexity and Motor-Relatedness was significant. Simple cognate motor verbs elicited more activation than simple cognate non-motor verbs $(t=2.50, p<.05)$, whereas complex motor and non-motor verbs showed a trend in the opposite direction $(t=-1.92, p=.071)$. L2 speakers, however, showed no effects or interactions involving Motor-Relatedness, as with the S2 ROI. These findings are discussed in more detail in Section 4.

\subsubsection{Whole-brain analysis}

Full-factorial whole-brain analyses were done over both groups and for each group separately to investigate whether other brain areas showed increased activation for simple and/or complex cognate motor verbs compared to non-motor verbs. 


\subsubsection{Analysis over both groups}

3.4.2.1.1. Interaction between Complexity and Motor-Relatedness. In the Cognate Verb analysis over both groups, the [(Simple Cognate (Motor-Non-Motor))-(Complex (Motor-Non-Motor))] contrast revealed significant clusters of activation in bilateral somatosensory and motor areas, reaching from the postcentral gyrus to the central sulcus (Fig. 2).

With the reverse contrast, no significant clusters were found. 3.4.2.1.2. Simple effects. In the [Simple Cognate (Motor-NonMotor)] contrast, the same areas were activated as in the interaction contrast [(Simple Cognate (Motor-Non-Motor))-(Complex (Motor-Non-Motor))], in addition to other areas. Bilaterally, a significant cluster was found stretching from the postcentral gyrus to the central sulcus, extending into the rolandic operculum and the right precentral gyrus. Another bilateral cluster was located in the transverse temporal gyrus. In the right hemisphere, significant clusters were found in the amygdala and in the corpus callosum.
No significant clusters were found for the reverse contrast, nor for the contrasts between Complex Motor and Non-Motor verbs (i.e. [Complex (Motor-Non-Motor)] and [Complex (Non-Motor-Motor)]).

3.4.2.1.3. Interaction between Complexity, Motor-Relatedness and Language. The contrasts for the triple interaction yielded no significant clusters in either direction.

\subsubsection{Analysis of the $L 1$ group}

3.4.2.2.1. Interaction between Complexity and Motor-Relatedness. With the Cognate Verb analysis limited to L1 speakers, the [(Simple Cognate (Motor-Non-Motor))-(Complex (Motor-Non-Motor))] contrast yielded no significant results. However, when the $p<.005 /$ $k=65$ threshold was used, a left-hemisphere cluster of activation was found in the transverse temporal gyrus, and right-hemisphere clusters were seen in the claustrum, reaching into the putamen, and in the corpus callosum (Fig. 5).

Table 9

Pre/M ROI: Cognate Verb ANOVA on contrast values for cognate verbs for L1 and L2 speakers.

\begin{tabular}{|c|c|c|c|c|c|c|}
\hline Effect & \multicolumn{2}{|c|}{ Both groups } & \multicolumn{2}{|c|}{ L1 speakers } & \multicolumn{2}{|c|}{ L2 speakers } \\
\hline Complexity & .59 & .45 & 5.58 & .029 & 2.89 & .11 \\
\hline Complexity $\times$ Motor & 5.44 & .025 & 9.95 & .005 & .36 & .56 \\
\hline Language & .98 & .33 & NA & NA & NA & NA \\
\hline Complexity $\times$ Language & 8.21 & .007 & NA & NA & NA & NA \\
\hline Motor $\times$ Language & .02 & .89 & NA & NA & NA & NA \\
\hline
\end{tabular}

Notes: df: Both Groups: 1,36; L1 speakers: 1,19; L2 speakers: 1,17. Motor: Motor-Relatedness. NA: Not applicable.

\section{Cognate Verbs}

b

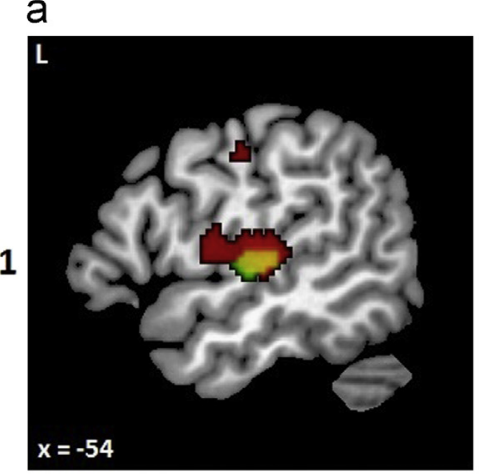

d

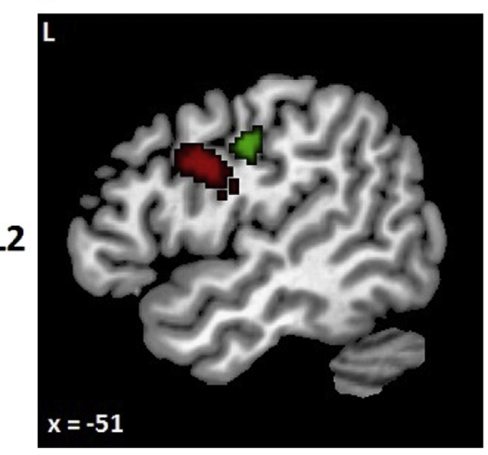

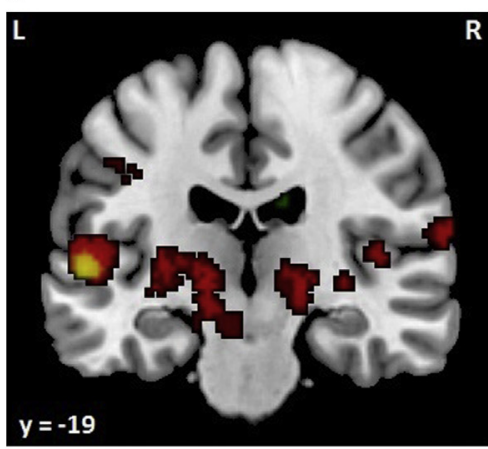

e

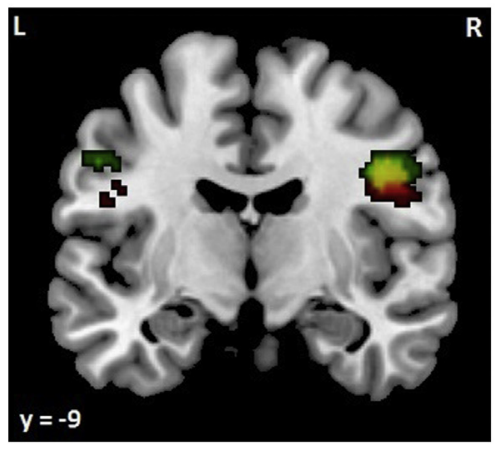

C

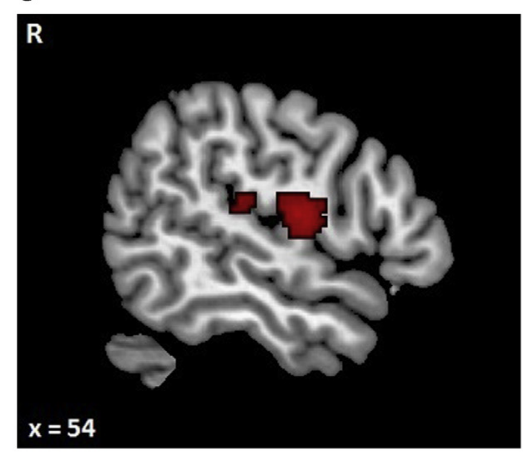

$f$

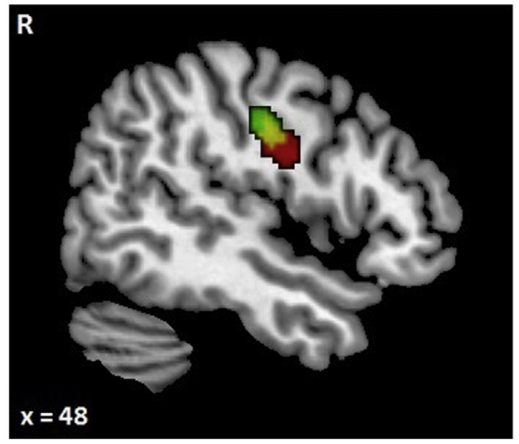

$\square$ Simple Cognate (Motor - Non-Motor) $\square$ Interaction Motor*Complexity $\square$ Overlap

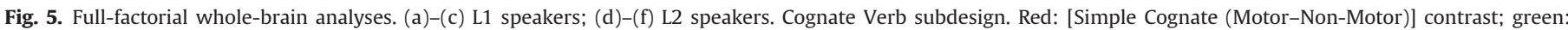

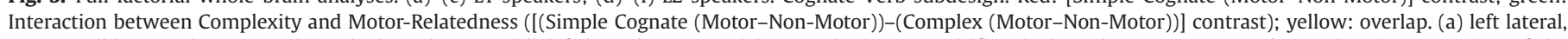

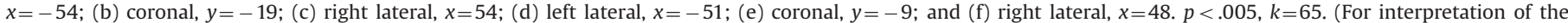
references to color in this figure legend, the reader is referred to the web version of this article.) 
The reverse contrast did not yield any significant clusters, neither at the $p<.001 / k=43$ nor at the $p<.005 / k=65$ threshold.

3.4.2.2.2. Simple effects. The [Simple Cognate (Motor-NonMotor)] contrast did yield significant clusters of activation at the $p<.001 / k=43$ threshold. One significant cluster was found in the left transverse temporal gyrus, overlapping with the cluster seen in the same region for the contrast for the Complexity by MotorRelatedness interaction. In the right hemisphere, a significant cluster was found in the rolandic operculum. Other clusters were seen in the left thalamus, and from the bilateral putamen, claustrum and insula to the left amygdala.

The reverse contrast did not yield any significant clusters, neither at the $p<.001 / k=43$ nor at the $p<.005 / k=65$ threshold. The same holds for the contrasts between Complex Motor and Non-Motor verbs.

\subsubsection{Analysis of the L2 group}

3.4.2.3.1. Interaction between Complexity and Motor-Relatedness. As with L1 speakers, the Cognate Verb analysis with L2 speakers revealed no significant results for the [(Simple Cognate (Motor-Non-Motor))-(Complex (Motor-Non-Motor))] contrast. With the $p<.005 / k=65$ threshold, however, clusters of activation were revealed in somatosensory and motor areas, i.e. from the bilateral postcentral gyrus to the central sulcus, reaching into the right precentral gyrus (Fig. 5).

The reverse contrast yielded no significant results with either threshold.

3.4.2.3.2. Simple effects. Contrasting Simple Cognate Motor and Non-Motor verbs revealed a significant cluster in the left anterior cingulate cortex, extending into the medial frontal gyrus. With the $p<.005 / k=65$ threshold, additional clusters of activation were found in somatosensory and motor areas, i.e. from the bilateral central sulcus and precentral gyrus into the right postcentral gyrus. The righthemisphere cluster overlapped with the right-hemisphere activation found for the Complexity by Motor-Relatedness interaction (Fig. 5).

The reverse contrast showed clusters in the right angular gyrus with both thresholds, and in the corpus callosum, reaching into the ventral anterior cingulate gyrus, with the $p<.005 / k=65$ threshold.

Finally, the [Complex (Motor-Non-Motor)] contrast did not reveal any significant activations for L2 speakers with either threshold, whereas the reverse contrast showed left-hemisphere clusters of activation stretching from the medial to the superior frontal gyrus, and from the ventral anterior cingulate cortex to the rostral gyrus, but only with the $p<.005 / k=65$ threshold.

3.4.2.4. Summary. To summarize, bilateral somatosensory and motor areas (postcentral gyrus to central sulcus) were significantly activated in the analysis over both groups for the [Simple Cognate (Motor-Non-Motor)] contrast as well as for the interaction between Complexity and Motor-Relatedness [(Simple Cognate (Motor-Non-Motor))-(Complex (Motor-Non-Motor))]. The triple interaction between Complexity, Motor-Relatedness and Language revealed no significant results.

When the two groups were analyzed separately, the Complexity by Motor-Relatedness interaction only yielded significant results with the $p<.005 / k=65$ threshold. In addition, the clusters of activation for this interaction were in slightly different locations for the two groups: in the left transverse temporal gyrus and right rolandic operculum for the L1 speakers, and in the bilateral postcentral gyrus and central sulcus for the L2 speakers (see Appendix E in Supplementary Materials). These differences are discussed below. For both groups, the clusters for the Complexity by Motor-Relatedness interaction overlapped with the clusters found when contrasting Simple Cognate Motor and Non-Motor conditions.

No significant clusters were found for the [Complex (MotorNon-Motor)] contrast in any analysis.

\section{Discussion}

The central question of the present study was whether embodiment effects are obtained not only for L1 speakers, replicating earlier studies, but also for L2 speakers. We studied both simple verbs and opaque complex verbs. The Simple Verb subdesign revealed a significant main effect of Motor-Relatedness, both in the ROI analyses and the whole-brain analysis over both groups. Simple motor verbs elicited higher activation than simple nonmotor verbs in motor and somatosensory regions. When the L1 and L2 participants were analyzed separately, similar patterns were found, indicating that embodiment effects with simple verbs were present with both groups. Crucially, L2 participants showed no interaction between Cognate Status and Motor-Relatedness in the regions of interest, indicating that embodiment effects with simple verbs for L2 speakers were not primarily driven by cognate verbs. Thus, Rüschemeyer et al.'s (2007) results for simple verbs in German were replicated for L1 speakers of Dutch and extended to L2 speakers.

For the Cognate Verb subdesign, the results for L1 and L2 participants did not completely converge in the ROI analyses. L1 participants showed a significant or marginally significant interaction between Complexity and Motor-Relatedness in the Pre/M and the S2 ROI, respectively, and no significant interaction in the SM ROI, whereas L2 speakers showed no such interaction in any ROI. In contrast, the whole-brain analyses did reveal significant activations for this interaction contrast for both L1 and L2 speakers, albeit at slightly differing locations and with the less conservative threshold of $p<.005 / k=65$ (see below for a discussion of the differing localization). It should be noted, however, that the analyses over both groups revealed more robust Complexity by Motor-Relatedness interaction effects, with simple motor verbs eliciting more activation than simple non-motor verbs in somatosensory and motor regions, and no difference between complex motor and non-motor verbs. In addition, no triple interactions between Language, Complexity and Motor-Relatedness were found, neither in the ROI nor the whole-brain analyses over both groups. The results for both simple and complex verbs are discussed in more detail below.

\subsection{Simple Verb subdesign: embodiment effects with simple verbs}

Increased activation for simple motor versus non-motor verbs was found in all three motor- and/or somatosensory-related ROIs (S2, SM and Pre/M) in the analyses over both groups. In the wholebrain analysis over both groups, activations were found in motor and somatosensory regions, i.e. pre- and postcentral gyrus, central sulcus, and parietal operculum (S2). In line with these results, increased activation for simple motor verbs has been found in previous studies in motor and/or premotor cortex (Hauk et al., 2004; Kemmerer et al., 2008; Raposo et al., 2009; Rüschemeyer et al., 2007; but see Postle et al., 2008) and in S2 (Rüschemeyer et al., 2007).

Crucially, there were no clear indications of differences between L1 and L2 participants in terms of activations in motor and somatosensory areas for simple verbs: Firstly, the ROI analyses revealed no significant interactions involving Language and MotorRelatedness; secondly, in the whole-brain analysis, the areas showing significant activation for interactions involving these factors (Language by Motor-Relatedness interaction: left supramarginal gyrus and right calcarine fissure; Language by Cognate Status by Motor-Relatedness interaction: right putamen) did not overlap with the areas showing significant activation for the [Simple (Motor-Non-Motor)] contrast.

Separate analyses of L1 and L2 participants showed similar patterns. In the ROI analyses, simple motor verbs elicited more 
activation than simple non-motor verbs in both groups, although not all differences reached significance: In the SM ROI, the main effect of Motor-Relatedness just missed significance for L1 speakers $(p=.053)$; in the Pre/M ROI, this effect did not reach significance for L2 speakers $(p=.11)$. A possible reason for these results is that the SM and Pre/M ROIs do not capture embodiment effects with our participants well enough because they are based on coordinates from another study (Rüschemeyer et al., 2007). Fig. 1 supports this suggestion: It shows that there is only a slight overlap between the Pre/M ROI and the areas significantly activated in the whole-brain analysis, and no overlap when the SM ROI is considered. This contrasts with the S2 ROI, which does show full overlap with these areas, and which displays robust embodiment effects with simple verbs in the separate ROI analyses for both L1 and L2 participants.

Regarding the results for the S2 ROI in L2 speakers, it should be noted that the increased activation for simple non-cognate motor versus non-motor verbs in this ROI is due to differences in deactivation rather than positive activation. It is difficult, however, to interpret contrast values for individual conditions below baseline. Possibly, the baseline is different in L1 and L2 speakers, but we do not have an obvious explanation for differences in baseline between the two groups. ${ }^{3}$ Potential baseline issues make it hard to interpret deactivations. Therefore, we think it is safer to interpret only differences in contrast values between different experimental conditions, rather than interpreting contrast values of individual conditions.

Like the ROI analyses, the whole-brain analyses for the two groups separately show quite similar patterns for the two groups. Significant activations for simple motor compared to non-motor verbs were found in somatosensory and motor-related regions in both groups, i.e. bilateral S2, and central sulcus, pre- and postcentral gyri in the right hemisphere. L1 speakers also showed significant activation in the left central sulcus and postcentral gyrus. To find out whether the lack of activation in the latter regions with $\mathrm{L} 2$ speakers was related to thresholding issues, we tested the [Simple (Motor-Non-Motor)] contrast at the $p=.005 /$ $k=65$ threshold. With this threshold, L2 participants did show a significant left-hemisphere cluster stretching from the precentral gyrus over the central sulcus to the postcentral gyrus. In addition, in the whole-brain analysis over both groups, the contrasts for the interaction involving Language and Motor-Relatedness did not reveal any activation in this area, neither at the $p=.001 / k=43$ nor at the $p=.005 / k=65$ threshold. Together, these findings argue against a difference between L1 and L2 participants regarding activations in motor and somatosensory areas for simple verbs.

Importantly, the embodiment effects with simple verbs do not seem to be merely driven by cognate verbs, i.e. by items for which L1 co-activation is most likely. As expected, for L1 speakers, neither ROI nor whole-brain analyses revealed significant interactions between Cognate Status and Motor-Relatedness, ruling out any systematic relevant differences between the two groups of verbs apart from cognate status. Crucially, for L2 speakers, the same was true for the ROI analyses. The whole-brain analysis for L2 speakers did show Cognate Status by Motor-Relatedness

\footnotetext{
${ }^{3}$ That this may be a baseline issue, is supported by the results of an alternative analysis of the S2 ROI we conducted for L2 speakers. For this, we used contrast values for each condition compared to the implicit baseline, rather than to the null condition. Results showed positive activation levels for both simple cognate and simple non-cognate motor verbs. A repeated-measures ANOVA on the Simple Verb subdesign (including the factors of Cognate Status (Cognate vs. Non-Cognate) and Motor-Relatedness (Motor vs. Non-Motor)) indicated that the main effect of MotorRelatedness was significant $(F(1,17)=11.13, p<.01)$, whereas the Cognate Status by Motor-Relatedness interaction was not $(F(1,17)=1.95, p=.18)$. These results indicate that, with a different baseline, simple non-cognate motor verbs do exhibit positive activation in the S2 ROI with L2 speakers.
}

interactions, but a follow-up analysis contrasting simple noncognate motor and non-motor verbs indicated that somatosensory and motor regions are activated even when cognate verbs are not included in the analysis. In addition, the regions showing increased activation in the interaction contrasts are associated with executive control and decision-related processes, not overlapping with the sensorimotor areas activated in the [Simple (Motor-Non-Motor)] contrast. Possibly, the difficulty of the L2 lexical decision task and the consequent increase in reaction times allows an interaction between motor-related and decision-related processing to develop: Activation of motor-related areas with simple motor (hand-related) verbs may prime the manual button press, thus interfering with the decision process (to press or not to press).

The two interaction contrasts showed activations in different executive control regions. More specifically, the [(Simple Cognate (Motor-Non-Motor))-(Simple Non-Cognate (Motor-Non-Motor))] contrast revealed significant activations in left caudal anterior cingulate cortex (CACC) reaching into the medial and superior frontal gyri (M/SFG), and in the left inferior precentral sulcus (iPCS). A network involving the left cACC and M/SFG has been implicated in cognitive control processes, i.e. monitoring and implementing cognitive control, for example in situations of response conflict (Aarts, 2009; Bush, Luu, \& Posner, 2000; Koski \& Paus, 2000; Paus, 2001; Ridderinkhof, Ullsperger, Crone, \& Nieuwenhuis, 2004). Similarly, the left iPCS has been associated with cognitive control processes, i.e. the activation and updating of task(-relevant) representations, for example in task-switching contexts or in the Stroop task (Brass, Derrfuss, Forstmann, \& von Cramon, 2005; Bunge, Kahn, Wallis, Miller, \& Wagner, 2003; Derrfuss, Brass, Neumann, \& von Cramon, 2005; Derrfuss, Vogt, Fiebach, von Cramon, \& Tittgemeyer, 2012). It should be added, though, that activation in the left iPCS has also been found as a reflection of motor-related processing (Grèzes \& Decety, 2001; Mayka, Corcos, Leurgans, \& Vaillancourt, 2006; Rizzolatti, Fogassi, \& Gallese, 2002). However, we did not find a similar activation for L1 speakers for simple motor versus non-motor verbs, which we would have expected if the left iPCS activation was motor-related. Also, the left iPCS activation in L2 speakers is accompanied by increased activation in other executive control regions (see above). Therefore, it seems likely that the left iPCS activation in L2 speakers reflects executive control rather than motor-related processing.

The reverse interaction contrast [(Simple Non-Cognate (MotorNon-Motor))-(Simple Cognate (Motor-Non-Motor))] showed a more anteriorly and ventrally located activation, in ventral ACC and ventromedial prefrontal cortex. These areas have been implicated in cognitively demanding tasks eliciting performance anxiety (Bush et al., 2000; Paus, 2001; Simpson, Drevets, Snyder, Gusnard, \& Raichle, 2001; Simpson, Snyder, Gusnard, \& Raichle, 2001).

The caudal versus ventral localization of ACC/prefrontal activations for cognate versus non-cognate motor verbs, respectively, suggests that, for L2 speakers, different types of decision processes may be involved depending on the cognate status of the verb. This is plausible, given that cognates, as opposed to non-cognates, are formally very similar to their translation equivalents. As has been shown in many behavioral studies (e.g., de Groot, Borgwaldt, Bos, \& van den Eijnden, 2002; Dijkstra et al., 2010; Lemhöfer \& Dijkstra, 2004), L2 speakers process cognates and non-cognates differently in deciding whether they are indeed L2 words. For L1 speakers, the difference in cognate status of L1 words (relative to L2 words) should not play a role in an L1 lexical decision task. This is supported by the lack of significant interactions involving Cognate Status with L1 speakers in this study.

The interaction involving L2 non-cognate motor verbs (the [(Simple Non-Cognate (Motor-Non-Motor))-(Simple Cognate (Motor-Non-Motor))] contrast) also showed increased activation in the right angular gyrus. Further analyses indicate that this 
activation (as opposed to the activations described above) is due to increased activation for simple cognate non-motor versus motor verbs, rather than to simple non-cognate motor versus non-motor verbs. We have no obvious interpretation for this activation. At first sight, one could think that it is related to abstract versus concrete word processing: The angular gyrus has been associated with complex semantic processing, including concept retrieval (Binder et al., 2009), and our simple non-motor stimuli may be more abstract than our simple motor stimuli. However, this explanation is contradicted by studies showing increased activation in the angular gyrus for concrete versus abstract words, rather than the reverse pattern (Binder et al., 2009). Therefore, it is not possible to use the concrete versus abstract dimension as a possible explanation.

Our interpretation that the activations found in both L1 and L2 speakers for simple motor versus non-motor verbs are due to their difference in motor-related characteristics is supported by our behavioral results and ratings. The error rates to simple verbs showed no significant effects or interactions involving MotorRelatedness in either group. This indicates that the embodiment effects we found for both groups cannot be explained by differences in cognitive load between simple motor and non-motor verbs. In addition, simple motor and non-motor verbs were matched for a number of variables, i.e. length and frequency. They did differ in terms of motor-relatedness, which was not only established by independent raters in a pretest, but also confirmed by the fMRI participants in an off-line rating following the fMRI experiment. Thus, the fMRI results can be ascribed to motorrelated characteristics of our stimuli.

The finding that simple verbs elicit activations in sensorimotor areas in L2 speakers has several implications for L2 processing models. Firstly, both the RHM and the BIA + models hypothesize that semantic representations are shared in L1 and L2 (Dijkstra \& Van Heuven, 2002; Kroll \& Stewart, 1994). This led to the prediction that similar motor-related activations should be generated in L1 and L2 speakers. Our results are in accordance with this prediction. Secondly, the Sense Model postulates that L2 semantic representations are less rich than L1 representations (Finkbeiner et al., 2004). However, our results suggest that $\mathrm{L} 2$ representations are rich enough to generate similar motor-related activations as L1 representations. The finding that both cognate and non-cognate verbs elicit embodiment effects indicates that this is not just due to transfer from L1 to L2 in L2 speakers.

Possibly, the similarity between L1 and L2 embodiment effects may be due to the high proficiency of the participants we tested. With less proficient L2 speakers, differences in terms of embodiment might have arisen (Bergen et al., 2010; Vukovic, 2012). This effect of proficiency may also have been enhanced by the immersion of our L2 speakers in an L2 environment.

\subsection{Cognate Verb subdesign: holistic processing of opaque complex verbs?}

No embodiment effects were found to semantically opaque complex verbs, as opposed to simple verbs, in any of the ROI or whole-brain analyses. The Complexity by Motor-Relatedness interaction over both groups revealed significant activations in sensorimotor areas, stretching bilaterally from the postcentral gyrus to the central sulcus. These regions were encompassed within the areas activated by the [Simple Cognate (Motor-Non-Motor)] contrast. The lack of significant activations for the [Complex (MotorNon-Motor)] contrast indicates that the meaning of the (motor) stem of opaque complex verbs was not accessed, suggesting that opaque complex verbs are not decomposed but processed holistically, in line with Rüschemeyer et al.'s (2007) study.
Of crucial interest to our study is the comparison between L1 and L2 speakers. The analyses over both groups give no indications that L1 and L2 speakers display differences with regard to embodiment effects with simple and complex cognate verbs. None of the ROI analyses reveal interactions involving Language and Motor-Relatedness, and the whole-brain analysis over both groups does not reveal significant activations for the triple interaction between Language, Complexity, and Motor-Relatedness.

In the ROI analyses of the two groups separately, L1 and L2 speakers also show a similar pattern descriptively, i.e. more activation for motor- than non-motor-related verbs with simple cognate verbs, but not with complex verbs. However, the Complexity by Motor-Relatedness interaction is not always significant. In L1 participants, an interaction is found, but less robustly than in the analyses over both groups: It is significant in the Pre/M ROI and marginally significant in the S2 ROI, but absent in the SM ROI. In L2 participants, none of the ROI analyses reveal significant Complexity by Motor-Relatedness interactions.

The less robust ROI results for the interaction with the two groups separately may again be related to the suboptimal location of the SM and Pre/M ROIs with regard to our participants: The overlap between these ROIs and activations in the whole-brain analyses is small, possibly because the ROIs are based on coordinates from another study (Rüschemeyer et al., 2007). This may make it more difficult to detect significant interactions in these ROIs.

In the whole-brain analyses of the two groups separately, the Complexity by Motor-Relatedness interaction is also less robust than in the whole-brain analysis over both groups. For either group, the Complexity by Motor-Relatedness interaction only reveals significant activations when the threshold is changed. In addition, the pattern of activation differs between L1 and L2 speakers. L1 participants show a cluster of activation in the left transverse temporal gyrus, whereas with L2 participants, activations are found in bilateral postcentral gyrus and central sulcus.

A possible reason for these differing localizations may be differences in language proficiency and/or native language influence for L2 compared to L1 participants: These may have led to between-group differences in the relative prominence of semantic components of the motor verbs presented (Kemmerer et al., 2008).

Notwithstanding these differences, both groups show significantly more activation for simple cognate motor than non-motor verbs in sensorimotor regions, which in each group encompass the regions found for the Complexity by Motor-Relatedness interaction. The processing of complex verbs is also very similar for the two groups: No differences in activation levels are found for complex motor compared to non-motor verbs in either group. Both these results replicate Rüschemeyer et al.'s (2007) findings for L1 speakers and extend them to L2 speakers. The results of the present study do differ from Rüschemeyer et al.'s results in one respect: For L1 speakers, the activation levels for complex verbs (both motor and non-motor complex verbs) are as high as for simple motor verbs in this study in most sensorimotor regions (see Figs. 2 and 3). This is not the case in Rüschemeyer et al.'s study, in which activation levels for complex verbs were in between those for simple motor and non-motor verbs. However, a direct comparison of simple and complex verbs cannot be interpreted in any straightforward way, as it was not possible to match these verbs on characteristics such as word length and frequency: Complex verbs were longer and less frequent than simple verbs (see Appendix B in Supplementary Materials).

The lack of differences in activation levels for complex motor compared to non-motor verbs in either group suggests that the (motor-related) meaning of the stem of opaque complex (motor) verbs is not accessed. These results are in agreement with many behavioral priming studies showing a lack of priming for morphologically complex, semantically opaque words (Feldman \& Soltano, 
1999; Feldman et al., 2002, 2004; Gonnerman et al., 2007; Longtin et al., 2003; Marslen-Wilson et al., 1994; Rastle et al., 2000; Zwitserlood et al., 2005). However, in several morphological priming studies (Luttmann et al., 2011; Smolka et al., 2009), German opaque complex verbs did give evidence of being decomposed by native speakers of German. One could hypothesize that this discrepancy is due to the task being used: Perhaps decomposition of opaque complex verbs was induced by the morphological priming technique (in which, e.g., a prime like reheat is followed by a target like heat) used in these studies.

\section{Conclusion}

In this study, we found evidence of language embodiment in L1 speakers as well as advanced L2 speakers: Both groups displayed higher activation for motor than for non-motor simple verbs in motor and somatosensory brain areas. These activations were evident with both cognate and non-cognate verbs, indicating that they were not due to transfer from the native language of the L2 speakers. In L2 speakers, additional activations in the ACC and prefrontal areas may have originated in differing decision processes for cognate and non-cognate verbs.

In contrast with simple verbs, opaque complex verbs did not show any evidence of language embodiment in either L2 or L1 speakers, as no increased activation was found for opaque complex motor versus non-motor verbs in any brain area. Thus, we found no evidence for decomposition of opaque complex verbs in either group, which suggests that these verbs are processed holistically. However, as the Complexity by Motor-Relatedness interaction did not reach significance in some of the analyses, this interpretation should be treated with caution. Future studies should investigate whether complex verbs are processed differently in different contexts. For example, motor-related activations for complex verbs might occur in a morphological priming context, as opposed to the unprimed presentation we used.

In summary, the present results show that L2 semantic representations are rich enough to lead to similar motor-related activations as in L1, and this embodiment effect is not restricted to cognate verbs, but also shows up for non-cognate verbs.

\section{Acknowledgments}

We are very grateful to Claudia Danielmeier and Jan Derrfuss for their advice on the imaging data. In addition, we would like to thank Frank Leoné and Florian Krause for their suggestions on the analysis.

\section{Appendix A. Supplementary materials}

Supplementary data associated with this article can be found in the online version at http://dx.doi.org/10.1016/j.neuropsychologia. 2014.02.003.

\section{References}

Aarts, E. (2009). Resisting temptation: The role of the anterior cingulate cortex in adjusting cognitive control (unpublished Ph.D. thesis). Nijmegen: Radboud University Nijmegen.

Baayen, R. H., Piepenbrock, R., \& Gulikers, L. (1995). The CELEX lexical database [CDROM]. Linguistic data consortium. Philadelphia, PA: University of Pennsylvania.

Barsalou, L. W. (2008). Grounded cognition. Annual Review of Psychology, 59(1), 617-645, http://dx.doi.org/10.1146/annurev.psych.59.103006.093639.

Basnight-Brown, D. M., Chen, L., Hua, S., Kostić, A., \& Feldman, L. B. (2007) Monolingual and bilingual recognition of regular and irregular English verbs: Sensitivity to form similarity varies with first language experience. Journal of Memory and Language, 57(1), 65-80, http://dx.doi.org/10.1016/j.jml.2007. 03.001 .
Bergen, B., Lau, T.-T., Narayan, S., Stojanovic, D., \& Wheeler, K. (2010). Body part representations in verbal semantics. Memory \& Cognition, 38(7), 969-981, http: //dx.doi.org/10.3758/mc.38.7.969.

Binder, J. R., Desai, R. H., Graves, W. W., \& Conant, L. L. (2009). Where is the semantic system? A critical review and meta-analysis of 120 functional neuroimaging studies. Cerebral Cortex, 19(12), 2767-2796, http://dx.doi.org/ 10.1093/cercor/bhp055.

Brass, M., Derrfuss, J., Forstmann, B., \& von Cramon, D. Y. (2005). The role of the inferior frontal junction area in cognitive control. Trends in Cognitive Sciences, 9 (7), 314-316, http://dx.doi.org/10.1016/j.tics.2005.05.001.

Brett, M., Anton, J. -L., Valabregue, R., \& Poline, J. -B. (2002). Region of interest analysis using the MarsBar toolbox for SPM 99 [abstract]. Paper presented at the 8th International conference on functional mapping of the human brain (Vol 16 (2) (Available on CD-ROM in Neurolmage).

Buchweitz, A., Shinkareva, S. V., Mason, R. A., Mitchell, T. M., \& Just, M. A. (2012). Identifying bilingual semantic neural representations across languages. Brain and Language, 120(3), 282-289, http://dx.doi.org/10.1016/j.bandl.2011.09.003.

Bunge, S. A., Kahn, I., Wallis, J. D., Miller, E. K., \& Wagner, A. D. (2003). Neural circuits subserving the retrieval and maintenance of abstract rules. Journal of Neurophysiology, 90(5), 3419-3428, http://dx.doi.org/10.1152/jn.00910.2002.

Bush, G., Luu, P., \& Posner, M. I. (2000). Cognitive and emotional influences in anterior cingulate cortex. Trends in Cognitive Sciences, 4(6), 215-222, http://dx. doi.org/10.1016/s1364-6613(00)01483-2.

Chee, M. W. L., Hon, N., Lee, H. L., \& Soon, C. S. (2001). Relative language proficiency modulates BOLD signal change when bilinguals perform semantic judgments. NeuroImage, 13(6), 1155-1163, http://dx.doi.org/10.1006/nimg.2001.0781.

Clahsen, H., \& Neubauer, K. (2010). Morphology, frequency, and the processing of derived words in native and non-native speakers. Lingua, 120(11), 2627-2637, http://dx.doi.org/10.1016/j.lingua.2010.06.007.

de Groot, A. M. B., Borgwaldt, S., Bos, M., \& van den Eijnden, E. (2002). Lexica decision and word naming in bilinguals: Language effects and task effects. Journal of Memory and Language, 47(1), 91-124, http://dx.doi.org/10.1006/ jmla.2001.2840.

Derrfuss, J., Brass, M., Neumann, J., \& von Cramon, D. Y. (2005). Involvement of the inferior frontal junction in cognitive control: Meta-analyses of switching and Stroop studies. Human Brain Mapping, 25(1), 22-34, http://dx.doi.org/10.1002/ hbm.20127.

Derrfuss, J., Vogt, V. L., Fiebach, C. J., von Cramon, D. Y., \& Tittgemeyer, M. (2012) Functional organization of the left inferior precentral sulcus: Dissociating the inferior frontal eye field and the inferior frontal junction. NeuroImage, 59(4), 3829-3837, http://dx.doi.org/10.1016/j.neuroimage.2011.11.051.

Diependaele, K., Duñabeitia, J. A., Morris, J., \& Keuleers, E. (2011). Fast morphological effects in first and second language word recognition. Journal of Memory and Language, 64(4), 344-358, http://dx.doi.org/10.1016/j.jml.2011.01.003.

Dijkstra, T., Miwa, K., Brummelhuis, B., Sappelli, M., \& Baayen, H. (2010). How crosslanguage similarity and task demands affect cognate recognition. Journal of Memory and Language, 62(3), 284-301, http://dx.doi.org/10.1016/j.jml.2009. 12.003.

Dijkstra, T., \& Van Heuven, W. J. B. (2002). The architecture of the bilingual word recognition system: From identification to decision. Bilingualism: Language and Cognition, 5(3), 175-197, http://dx.doi.org/10.1017/S1366728902003012.

Feldman, L. B., Barac-Cikoja, D., \& Kostić, A. (2002). Semantic aspects of morphological processing: Transparency effects in Serbian. Memory E Cognition, 30(4) 629-636, http://dx.doi.org/10.3758/bf03194964.

Feldman, L. B., \& Soltano, E. G. (1999). Morphological priming: The role of prime duration, semantic transparency, and affix position. Brain and Language, 68(12), 33-39, http://dx.doi.org/10.1006/brln.1999.2077.

Feldman, L. B. Soltano, E. G., Pastizzo, M. J., \& Francis, S. E. (2004). What do graded effects of semantic transparency reveal about morphological processing? Brain and Language, 90(1-3), 17-30, http://dx.doi.org/10.1016/s0093-934x(03)00416-4.

Finkbeiner, M., Forster, K., Nicol, J., \& Nakamura, K. (2004). The role of polysemy in masked semantic and translation priming. Journal of Memory and Language, 51 (1), 1-22, http://dx.doi.org/10.1016/j.jml.2004.01.004.

Forman, S. D., Cohen, J. D., Fitzgerald, M., Eddy, W. F., Mintun, M. A., \& Noll, D. C. (1995). Improved assessment of significant activation in functional magnetic resonance imaging (fMRI): Use of a cluster-size threshold. Magnetic Resonance in Medicine, 33(5), 636-647, http://dx.doi.org/10.1002/mrm.1910330508.

Gonnerman, L. M., Seidenberg, M. S., \& Andersen, E. S. (2007). Graded semantic and phonological similarity effects in priming: Evidence for a distributed connectionist approach to morphology. Journal of Experimental Psychology: General, 136(2), 323-345, http://dx.doi.org/10.1037/0096-3445.136.2.323.

Grèzes, J., \& Decety, J. (2001). Functional anatomy of execution, mental simulation, observation, and verb generation of actions: A meta-analysis. Human Brain Mapping, 12(1), 1-19, http://dx.doi.org/10.1002/1097-0193(200101)12:1<1:: aid-hbm10 > 3.0.co;2-v

Hauk, O., Johnsrude, I., \& Pulvermüller, F. (2004). Somatotopic representation of action words in human motor and premotor cortex. Neuron, 41(2), 301-307, http://dx.doi.org/10.1016/s0896-6273(03)00838-9.

Illes, J., Francis, W. S., Desmond, J. E., Gabrieli, J. D. E., Glover, G. H., Poldrack, R., et al. (1999). Convergent cortical representation of semantic processing in bilinguals Brain and Language, 70(3), 347-363, http://dx.doi.org/10.1006/brln.1999.2186.

Isel, F., Baumgaertner, A., Thrän, J., Meisel, J. M., \& Büchel, C. (2010). Neural circuitry of the bilingual mental lexicon: Effect of age of second language acquisition. Brain and Cognition, 72(2), 169-180, http://dx.doi.org/10.1016/j.bandc.2009. 07.008 . 
Kemmerer, D., Castillo, J. G., Talavage, T., Patterson, S., \& Wiley, C. (2008) Neuroanatomical distribution of five semantic components of verbs: Evidence from fMRI. Brain and Language, 107(1), 16-43, http://dx.doi.org/10.1016/j. bandl.2007.09.003.

Koski, L., \& Paus, T. (2000). Functional connectivity of the anterior cingulate cortex within the human frontal lobe: A brain-mapping meta-analysis. Experimental Brain Research, 133(1), 55-65, http://dx.doi.org/10.1007/s002210000400.

Kroll, J. F., \& Stewart, E. (1994). Category interference in translation and picture naming: Evidence for asymmetric connections between bilingual memory representations. Journal of Memory and Language, 33(2), 149-174, http://dx doi.org/10.1006/jmla.1994.1008.

Lehtonen, M., \& Laine, M. (2003). How word frequency affects morphological processing in monolinguals and bilinguals. Bilingualism: Language and Cognition, 6(3), 213-225, http://dx.doi.org/10.1017/S1366728903001147.

Lemhöfer, K., \& Broersma, M. (2012). Introducing LexTALE: A quick and valid lexical test for advanced learners of English. Behavior Research Methods, 44(2), 325-343, http://dx.doi.org/10.3758/s13428-011-0146-0.

Lemhöfer, K., \& Dijkstra, T. (2004). Recognizing cognates and interlingual homographs: Effects of code similarity in language-specific and generalized lexica decision. Memory \& Cognition, 32(4), 533-550, http://dx.doi.org/10.3758 bf03195845.

Lemhöfer, K., Koester, D., \& Schreuder, R. (2011). When bicycle pump is harder to read than bicycle bell: Effects of parsing cues in first and second language compound reading. Psychonomic Bulletin E' Review, 18(2), 364-370, http://dx. doi.org/10.3758/s13423-010-0044-y.

Longtin, C.-M., Segui, J., \& Hallé, P. A. (2003). Morphological priming without morphological relationship. Language and Cognitive Processes, 18(3), 313-334, http://dx.doi.org/10.1080/01690960244000036.

Luttmann, H., Zwitserlood, P., \& Bolte, J. (2011). Sharing morphemes without sharing meaning: Production and comprehension of German verbs in the context of morphological relatives. Canadian Journal of Experimental Psychology, 65(3), 173-191, http://dx.doi.org/10.1037/a0023794.

Mahon, B. Z., \& Caramazza, A. (2008). A critical look at the embodied cognition hypothesis and a new proposal for grounding conceptual content. Journal of Physiology-Paris, 102(1-3), 59-70, http://dx.doi.org/10.1016/j.jphysparis.2008. 03.004 .

Marslen-Wilson, W. D., Tyler, L. K., Waksler, R., \& Older, L. (1994). Morphology and meaning in the English mental lexicon. Psychological Review, 101(1) 3-33.

Mayka, M. A., Corcos, D. M., Leurgans, S. E., \& Vaillancourt, D. E. (2006). Threedimensional locations and boundaries of motor and premotor cortices as defined by functional brain imaging: A meta-analysis. Neurolmage, 31(4) 1453-1474, http://dx.doi.org/10.1016/j.neuroimage.2006.02.004.

Neubauer, K., \& Clahsen, H. (2009). Decomposition of inflected words in a second language. Studies in Second Language Acquisition, 31, 403-435, http://dx.doi.org/ $10.1017 /$ S0272263109090354.

Noppeney, U., Josephs, O., Kiebel, S., Friston, K. J., \& Price, C. J. (2005). Action selectivity in parietal and temporal cortex. Cognitive Brain Research, 25(3) 641-649, http://dx.doi.org/10.1016/j.cogbrainres.2005.08.017.

Paus, T. (2001). Primate anterior cingulate cortex: Where motor control, drive and cognition interface. Nature Reviews Neuroscience, 2(6), 417-424, http://dx.doi. org/10.1038/35077500.

Portin, M., \& Laine, M. (2001). Processing cost associated with inflectional morphology in bilingual speakers. Bilingualism: Language and Cognition, 4(1) 55-62, http://dx.doi.org/10.1017/S1366728901000128.

Portin, M., Lehtonen, M., Harrer, G., Wande, E., Niemi, J., \& Laine, M. (2008). L1 effects on the processing of inflected nouns in L2. Acta Psychologica, 128(3), 452-465, http://dx.doi.org/10.1016/j.actpsy.2007.07.003.

Postle, N., McMahon, K. L., Ashton, R., Meredith, M., \& de Zubicaray, G. I. (2008) Action word meaning representations in cytoarchitectonically defined primary and premotor cortices. NeuroImage, 43(3), 634-644, http://dx.doi.org/10.1016/j. neuroimage.2008.08.006.

Raposo, A., Moss, H. E., Stamatakis, E. A., \& Tyler, L. K. (2009). Modulation of motor and premotor cortices by actions, action words and action sentences.
Neuropsychologia, 47(2), 388-396, http://dx.doi.org/10.1016/j.neuropsychologia. 2008.09.017.

Rastle, K., Davis, M. H., Marslen-Wilson, W. D., \& Tyler, L. K. (2000). Morphological and semantic effects in visual word recognition: A time-course study. Language and Cognitive Processes, 15(4-5), 507-537, http://dx.doi.org/10.1080/01690960 050119689.

Ridderinkhof, K. R., Ullsperger, M., Crone, E. A., \& Nieuwenhuis, S. (2004). The role of the medial frontal cortex in cognitive control. Science, 306(5695), 443-447, http://dx.doi.org/10.1126/science.1100301.

Rizzolatti, G., Fogassi, L., \& Gallese, V. (2002). Motor and cognitive functions of the ventral premotor cortex. Current Opinion in Neurobiology, 12(2), 149-154, http: //dx.doi.org/10.1016/S0959-4388(02)00308-2.

Ruben, J., Schwiemann, J., Deuchert, M., Meyer, R., Krause, T., Curio, G., et al. (2001). Somatotopic organization of human secondary somatosensory cortex. Cerebral Cortex, 11(5), 463-473, http://dx.doi.org/10.1093/cercor/11.5.463.

Rüschemeyer, S.-A., Brass, M., \& Friederici, A. D. (2007). Comprehending prehending: Neural correlates of processing verbs with motor stems. Journal of Cognitive Neuroscience, 19(5), 855-865, http://dx.doi.org/10.1162/jocn.2007.19.5.855.

Rüschemeyer, S.-A., Zysset, S., \& Friederici, A. D. (2006). Native and non-native reading of sentences: An fMRI experiment. NeuroImage, 31(1), 354-365, http: //dx.doi.org/10.1016/j.neuroimage.2005.11.047.

Silva, R., \& Clahsen, H. (2008). Morphologically complex words in L1 and L2 processing: Evidence from masked priming experiments in English. Bilingualism: Language and Cognition, 11(2), 245-260, http://dx.doi.org/10.1017/ S1366728908003404.

Simpson, J. R., Drevets, W. C., Snyder, A. Z., Gusnard, D. A., \& Raichle, M. E. (2001) Emotion-induced changes in human medial prefrontal cortex: II. During anticipatory anxiety. Proceedings of the National Academy of Sciences, 98(2), 688-693, http://dx.doi.org/10.1073/pnas.98.2.688.

Simpson, J. R. Snyder, A. Z, Gusnard, D. A., \& Raichle, M. E. (2001). Emotioninduced changes in human medial prefrontal cortex: I. During cognitive task performance. Proceedings of the National Academy of Sciences, 98(2), 683-687, http://dx.doi.org/10.1073/pnas.98.2.683.

Slotnick, S. D., Moo, L. R., Segal, J. B., \& Hart, J., (2003). Distinct prefrontal cortex activity associated with item memory and source memory for visual shapes. Cognitive Brain Research, 17(1), 75-82, http://dx.doi.org/10.1016/s0926-6410 (03)00082-x.

Smolka, E., Komlósi, S., \& Rösler, F. (2009). When semantics means less than morphology: The processing of German prefixed verbs. Language and Cognitive Processes, 24(3), 337-375, http://dx.doi.org/10.1080/01690960802075497.

Vukovic, N. (2012). Sensorimotor processing of action verbs in second language speakers [abstract]. Paper presented at the Embodied and situated language processing conference.

Wartenburger, I., Heekeren, H. R., Abutalebi, J., Cappa, S. F., Villringer, A., \& Perani, D. (2003). Early setting of grammatical processing in the bilingual brain. Neuron, 37(1), 159-170, http://dx.doi.org/10.1016/s0896-6273(02)01150-9.

Willems, R. M., \& Casasanto, D. (2011). Flexibility in embodied language understanding. Frontiers in Psychology, 2(116), 1-11, http://dx.doi.org/10.3389/ fpsyg.2011.00116.

Willems, R. M., Toni, I., Hagoort, P., \& Casasanto, D. (2009). Neural dissociations between action verb understanding and motor imagery. Journal of Cognitive Neuroscience, 22(10), 2387-2400, http://dx.doi.org/10.1162/jocn.2009.21386.

Zwitserlood, P., Bolwiender, A., \& Drews, E. (2005). Priming morphologically complex verbs by sentence contexts: Effects of semantic transparency and ambiguity. Language and Cognitive Processes, 20(1-2), 395-415, http://dx.doi. org/10.1080/01690960444000160. 